\title{
Development of an RNAi therapeutic for alpha-1-antitrypsin liver disease
}

\author{
Christine I. Wooddell, ${ }^{1}$ Keith Blomenkamp, ${ }^{2}$ Ryan M. Peterson, ${ }^{1}$ Vladimir M. Subbotin, ${ }^{1}$ \\ Christian Schwabe, ${ }^{3}$ James Hamilton, ${ }^{4}$ Qili Chu, ${ }^{1}$ Dawn R. Christianson, ${ }^{4}$ Julia O. Hegge, ${ }^{1}$ John Kolbe, ${ }^{3}$ \\ Holly L. Hamilton, ${ }^{1}$ Maria F. Branca-Afrazi, ${ }^{1}$ Bruce D. Given, ${ }^{4}$ David L. Lewis, ${ }^{1}$ Edward Gane, ${ }^{3}$ \\ Steven B. Kanner, ${ }^{1}$ and Jeffrey H. Teckman ${ }^{5}$ \\ 'Arrowhead Pharmaceuticals, Madison, Wisconsin, USA. ${ }^{2}$ Department of Pediatrics, St. Louis University School of \\ Medicine, St. Louis, Missouri, USA. ${ }^{3}$ Auckland Clinical Studies, Auckland, New Zealand. ${ }^{4}$ Arrowhead Pharmaceuticals, \\ Pasadena, California, USA. ${ }^{5}$ Departments of Pediatrics and Biochemistry, St. Louis University School of Medicine, Cardinal \\ Glennon Children's Hospital, St. Louis, Missouri, USA.
}

The autosomal codominant genetic disorder alpha-1 antitrypsin (AAT) deficiency (AATD) causes pulmonary and liver disease. Individuals homozygous for the mutant $Z$ allele accumulate polymers of Z-AAT protein in hepatocytes, where AAT is primarily produced. This accumulation causes endoplasmic reticulum (ER) stress, oxidative stress, damage to mitochondria, and inflammation, leading to fibrosis, cirrhosis, and hepatocellular carcinoma. The magnitude of AAT reduction and duration of response from first-generation intravenously administered RNA interference (RNAi) therapeutic ARC-AAT and then with next-generation subcutaneously administered ARO-AAT were assessed by measuring AAT protein in serum of the PiZ transgenic mouse model and human volunteers. The impact of Z-AAT reduction by RNAi on liver disease phenotypes was evaluated in PiZ mice by measuring polymeric Z-AAT in the liver; expression of genes associated with fibrosis, autophagy, apoptosis, and redox regulation; inflammation; Z-AAT globule parameters; and tumor formation. Ultrastructure of the ER, mitochondria, and autophagosomes in hepatocytes was evaluated by electron microscopy. In mice, sustained RNAi treatment reduced hepatic Z-AAT polymer, restored ER and mitochondrial health, normalized expression of disease-associated genes, reduced inflammation, and prevented tumor formation. RNAi therapy holds promise for the treatment of patients with AATD-associated liver disease. ARO-AAT is currently in phase II/III clinical trials.

Conflict of interest: CIW, RMP, VMS, JH, QC, DRC, JOH, HLH, MFBA, BDG, DLL, and SBK are or were employees of Arrowhead Pharmaceuticals and may be stockholders. JHT is a consultant for Arrowhead. CS and JK performed work under contract with Arrowhead. EG has been an advisor and/or speaker for AbbVie, Gilead, Janssen, Novartis, Roche, and Merck.

Copyright: () 2020, American Society for Clinical Investigation.

Submitted: December 2, 2019

Accepted: April 30, 2020

Published: June 18, 2020

Reference information: /CI Insight. 2020;5(12):e135348.

https://doi.org/10.1172/jici.

insight.135348.

\section{Introduction}

Human alpha-1 antitrypsin (AAT) is encoded by the SERPINA1 gene and functions primarily as an inhibitor of neutrophil elastase. The highly abundant serum glycoprotein AAT is produced primarily in hepatocytes, and its expression is upregulated during tissue injury and inflammation. AAT deficiency (AATD) is an autosomal codominant genetic disease most commonly due to homozygosity for the $\mathrm{Z}$ allele (PiZZ), which has a single point mutation (Glu342Lys) that causes the Z-AAT protein to fold abnormally, secrete poorly, and homopolymerize $(1,2)$. Only $10 \%-15 \%$ of liver-produced Z-AAT is secreted, and the decreased AAT in serum increases the risk of early pulmonary disease in adults. The remainder of the Z-AAT protein is retained in the liver, where ordered polymers form and cause varying degrees of liver injury in children and adults. Liver disease may manifest as jaundice due to hepatitis and portal hypertension occurring in newborns and children, fibrosis and cirrhosis occurring at any time in life, and hepatocellular carcinoma (HCC) occurring most frequently between the ages of 50 and 64 (3-7). A recent University of Florida study of 94 adults with PiZZ without diagnosed liver disease found that approximately one-third had at least F2 liver fibrosis on biopsy, and the degree of fibrosis correlated with the amount of Z-AAT in the liver (8). Many individuals with AATD-related liver disease are unaware of their condition until signs or symptoms of decompensated cirrhosis occur.

Z-AAT accumulation in the liver leads to increased apoptosis and promotes proliferation of progenitor cells, inflammation, and fibrosis (9-11). The PiZ mouse harbors the human Z-AAT genomic transgene and recapitulates many aspects of human AATD-associated liver disease $(12,13)$, thus facilitating insights into this disease. The accumulated Z-AAT polymer induces an injury cascade including endoplasmic reticulum (ER) 
stress, oxidant stress, increased inflammation, and an increased rate of hepatocyte death and proliferation of progenitor cells $(11,14)$. The amount of Z-AAT protein within individual hepatocytes correlates with liver injury and the degree to which the apoptotic cascade is stimulated in the PiZ mouse model $(10,15)$. The resulting regeneration of hepatocytes is accompanied by focal areas of inflammatory infiltrates, a low-grade inflammation that over time leads to adenomas and HCC in 70\%-80\% of PiZ mice by 18 months of age $(16,17)$.

Incompletely folded and polymeric Z-AAT proteins in the liver are degraded by ER-associated degradation (ERAD) and by autophagy (2, 18-20). High expression of Z-AAT protein in human cell lines, in PiZ mice, and in AATD patient livers induces an autophagic response as the Z-AAT polymer accumulates in the rough ER (rER) cisternae, leading to their dilation $(19,21)$. When the ERAD pathway and autophagocytosis are unable to prevent accumulation of the Z-AAT polymer, globular inclusion bodies, "globules," are formed that are visualized microscopically with periodic acid-Schiff staining after diastase digestion (PAS-D). Electron-dense multilamellar autophagic vacuoles have been observed near and budding from dilated ER in human fibroblasts engineered to express Z-AAT, in human PiZZ AATD individuals with or without evidence of liver disease and in PiZ mouse liver (19).

Individuals who are null for AAT do not accumulate Z-AAT in hepatocytes or develop associated liver disease (22), leading to the hypothesis that reduction of disease-causing Z-AAT protein in the liver mediated by RNA interference (RNAi) could improve liver health and reduce long-term risk of cirrhosis and HCC. RNAi is a naturally occurring process by which cells cleave mRNA hybridized to the complementary antisense strand of a small interfering RNA or synthetic RNAi trigger (23).

The initial studies in this report used the intravenously injected RNAi-based therapeutic ARC-AAT, and subsequent studies used the subcutaneously administered RNAi therapeutic ARO-AAT. ARC-AAT consisted of a cholesterol-conjugated RNAi trigger (chol-RNAi) to selectively degrade AAT mRNA by RNAi and a melittin-derived peptide conjugated to $N$-acetylgalactosamine (NAG) formulated as the excipient EX1 to promote endosomal escape of the chol-RNAi in hepatocytes $(24,25)$. These components were coinjected intravenously.

Treatment with ARC-AAT yielded deep and durable reductions in serum AAT in normal human volunteers and in patients with PiZZ, but clinical trials of ARC-AAT were terminated early because of toxicity concerns related to EX1 identified at elevated dose levels in nonhuman primate (NHP) studies (25). We subsequently developed the RNAi therapeutic ARO-AAT, an RNAi trigger targeted to hepatocytes through conjugation of NAG via a linker, thereby not requiring EX1. ARO-AAT is administered subcutaneously and is anticipated to exhibit a high therapeutic index.

Although serum reduction of ARC-AAT in normal volunteers and patients was previously published, this is likely the first publication demonstrating the beneficial effects of RNAi therapeutics on the liver of the PiZ mouse model. Following RNAi treatment, reduction in the amount of AAT in the plasma or serum served as a measure of RNAi activity and a convenient tool to evaluate the duration of effect. Reduction of AAT mRNA in the liver confirmed knockdown (KD) of gene expression. Semiquantitative Western blotting was used to measure the disease-causing monomeric and polymeric Z-AAT protein in the liver. Histological methods were used to assess globules and inflammation in the liver. Treatment-mediated changes to the ultrastructure of PiZ hepatocytes were evaluated by electron microscopy (EM).

\section{Results}

$R N A i$ therapeutic $A R C-A A T$. Development of ARC-AAT is described in the supplementary materials and Supplemental Figures 1-4; supplemental material available online with this article; https://doi. org/10.1172/jci.insight.135348DS1. ARC-AAT consists of chol-RNAi, which is the active pharmaceutical ingredient (API), and EX1 at a 2:1 weight/weight ratio. Varied ratios of chol-RNAi/EX1 were used during development of ARC-AAT. In the absence of EX1, the chol-RNAi alone dosed as high as $24 \mathrm{mg} / \mathrm{kg}$ was unable to reduce AAT (Supplemental Figure 5).

$R N A$-mediated reduction of Z-AAT prevents progression of the AATD liver disease phenotype in young PiZ mice. The effects of repeat dosing with the ARC-AAT API and EX1 on plasma Z-AAT protein and on Z-AAT mRNA in the livers of PiZ mice are shown in Figure 1. Five-week-old male and female PiZ mice given 4 or 5 coinjections of $8 \mathrm{mg} / \mathrm{kg}$ ARC-AAT API and $8 \mathrm{mg} / \mathrm{kg}$ EX1 (RNAi-AAT + EX1) every other week maintained greater than $90 \%$ plasma Z-AAT protein reduction from the second injection to the end of the study (Figure 1A). Liver Z-AAT mRNA concomitantly was reduced by greater than $90 \%$ (Figure 1B). Results were similar in males and females. Plasma Z-AAT KD appeared metronomically controlled in mice injected every third week, although a cumulative effect by week 10 was apparent after repeat dosing (Figure 1A). 
Male PiZ mice (5 weeks old at baseline) were used to evaluate RNAi-mediated changes in the liver after 8 weeks of treatment. The disease phenotype is known to be more severe and more consistent in males (14). Mice were given 4 Q2W injections of $8 \mathrm{mg} / \mathrm{kg}$ API and $8 \mathrm{mg} / \mathrm{kg}$ EX1 (RNAi-AAT + EX1) or saline and euthanized 2 weeks after the final injection (when 13 weeks old). A control group was given 4 Q2W injections of $8 \mathrm{mg} / \mathrm{kg}$ chol-RNAi trigger to a nonmammalian sequence coinjected with $8 \mathrm{mg} /$ kg EX1 (RNAi-Control + EX1). Monomeric and polymeric Z-AAT protein in liver lysates of PiZ mice were separated by centrifugation to pellet the insoluble polymeric aggregates from the soluble monomeric Z-AAT; then both fractions were measured by semiquantitative Western blotting $(26,27)$. Relative to the age-matched saline control mice, monomeric Z-AAT protein in mice treated with RNAi-AAT + EX1 was $97 \% \pm 4 \%$ (SEM) lower, and polymeric Z-AAT was $79 \% \pm 10 \%$ lower (Figure $1, \mathrm{C}$ and D). Relative to baseline, monomer was reduced $90 \% \pm 6 \%$ and polymer $38 \% \pm 26 \%$.

As expected from the polymer quantitation, the size of globules containing Z-AAT polymer increased in livers of animals injected with saline or RNAi-Control + EX1, relative to baseline, but not in mice injected with RNAi-AAT + EX1 (Figure 1E). Treatment with RNAi-AAT + EX1 also appeared to prevent the modest infiltration of inflammatory cells that occurred in the 13-week-old saline-treated group and RNAi-Control + EX1 animals, but this change was not quantified.

$A R C$ - $A A T$ reduced preexisting $Z-A A T$ polymer and prevented inflammation in the liver in $P i Z$ mice. The highest numbers of globules in PiZ mouse hepatocytes have been observed in 3-month-old young adults (28). Male PiZ mice (11-17 weeks old at baseline) were treated with ARC-AAT to determine whether RNAi could reverse the liver disease phenotype (Figure 2). The mice were given Q2W IV injections of $8 \mathrm{mg} / \mathrm{kg}$ ARC-AAT ( $8 \mathrm{mg} / \mathrm{kg}$ API $+4 \mathrm{mg} / \mathrm{kg}$ EX1) for 30-31 weeks and evaluated 2 weeks after final injection. Some animals received an additional injection to maintain Z-AAT KD during a schedule adjustment, thus resulting in sacrifice at 33 rather than 32 weeks of treatment. Control groups were injected with saline, 12 $\mathrm{mg} / \mathrm{kg}$ API only, or $12 \mathrm{mg} / \mathrm{kg}$ RNAi-Control with $6 \mathrm{mg} / \mathrm{kg}$ EX1. Plasma was collected biweekly before reinjection for measurement of Z-AAT protein. Mice injected Q2W with ARC-AAT maintained 95\%-98\% mean reduction of plasma Z-AAT protein at each time point for the duration of treatment (Figure 2A). The reduction required ARC-AAT (API + EX1), whereas $12 \mathrm{mg} / \mathrm{kg}$ API alone did not reduce Z-AAT nor did RNAi-Control + EX1.

Monomeric Z-AAT protein in the livers of mice treated with ARC-AAT was reduced by $89 \%$, and polymeric Z-AAT was reduced $42 \%$, relative to baseline (Figure 2, B and C). In contrast, monomeric Z-AAT protein was unchanged, relative to baseline, but polymeric Z-AAT protein increased by $30 \%$ in the saline-injected group. The relative amounts of monomeric and polymeric Z-AAT in the RNAi-Control + EX1 and the API-alone groups were similar to the saline group (data not shown). Immunohistochemical detection of human AAT demonstrated that the Z-AAT polymer remaining after ARC-AAT treatment was in a globular form (Supplemental Figure 6).

Z-AAT globules were evaluated by PAS-D staining of liver specimens from PiZ mice at baseline or after 32/33 weeks of treatment (Figure 2, D-G). As determined by Western blotting (Figure 2, B and C) and PAS-D quantitation, a $42 \%$ reduction of globules was observed in ARC-AAT-treated mice relative to baseline. The number of globules was similarly reduced in mice of the same age that had been injected with only saline. Such an age-dependent decrease in globules was previously seen in PiZ mice (29). However, globules in the saline group animals were 2.75-fold larger and comprised 2.54-fold more area of the liver than those in mice treated with ARC-AAT (Figure 2, D, F, and G). The average diameter of globules at baseline was approximately $5-10 \mu \mathrm{m}$ and in the saline group 32/33 weeks later was approximately $20-50$ $\mu \mathrm{m}$. The area of the liver containing globules in mice treated with ARC-AAT was reduced by $61 \%$ relative to the age-matched saline group mice.

These results demonstrate that in the absence of RNAi intervention, polymeric Z-AAT continued to accumulate into much larger, though fewer, globules in the PiZ mouse liver over the course of 7-8 months while the amount of monomeric Z-AAT protein in the liver and in the plasma did not change. The mice treated with ARC-AAT had a small increase in the size of presumably preexisting globules. Z-AAT KD fluctuated during the study because of the technical difficulty of intravenous injection in darkly pigmented mice. It is unknown whether the periodically higher Z-AAT expression enabled globule growth.

Adult PiZ mice at baseline had small foci of inflammatory cells around dead hepatocytes in their livers (Figure 2, D, H, and I). These inflammatory foci consisted of a mixed infiltrate of granulocytes (mostly neutrophils) and monocytes (expected to be primarily macrophages, data not shown). At the end of the 


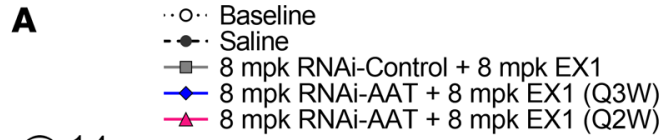

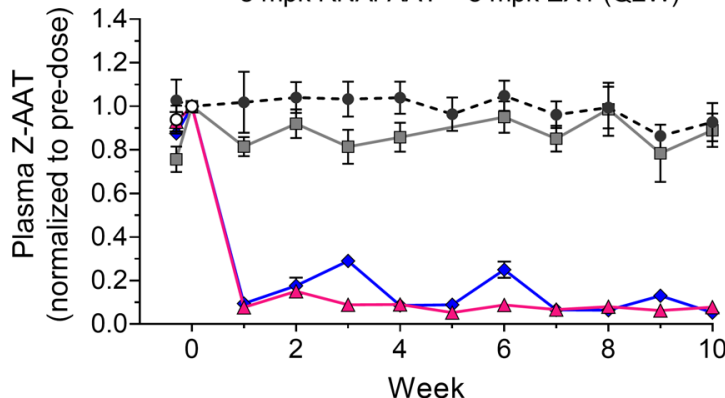

B

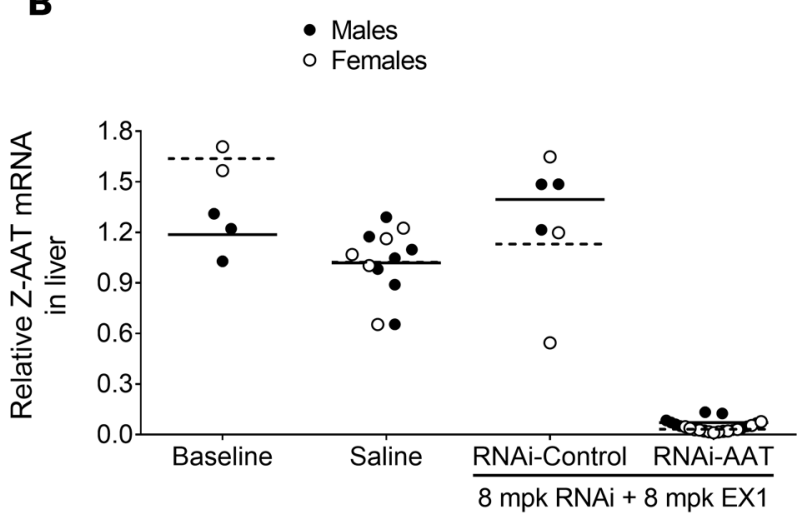

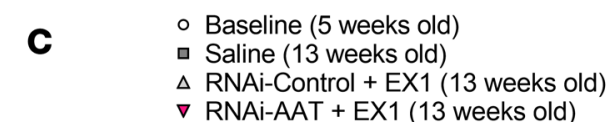

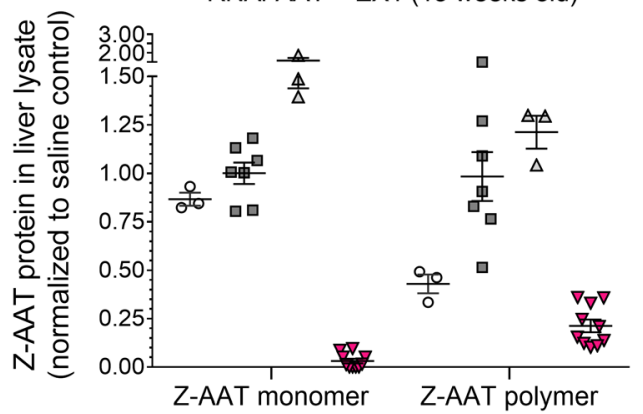

$\mathbf{E}$

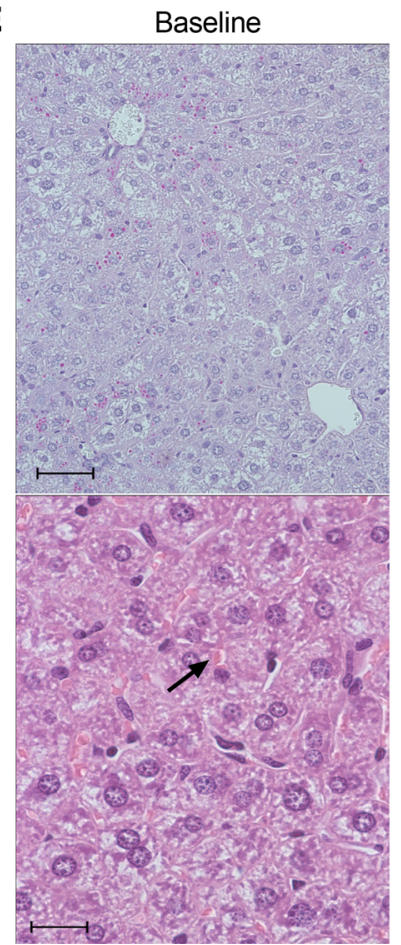

D

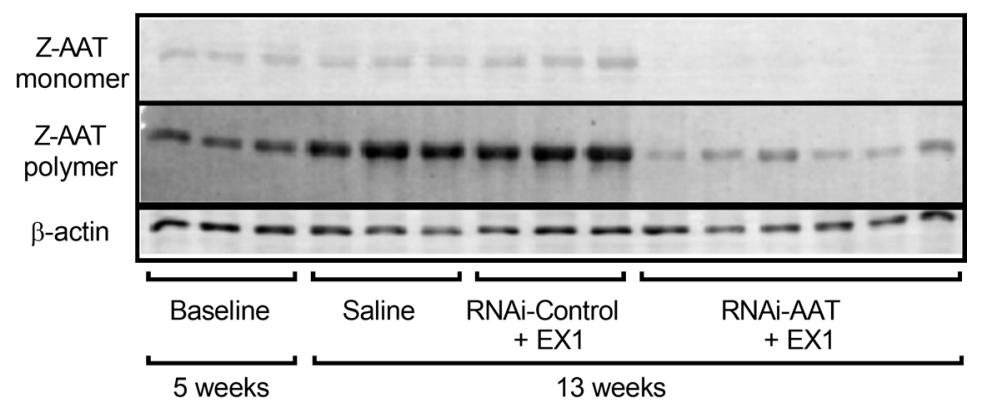

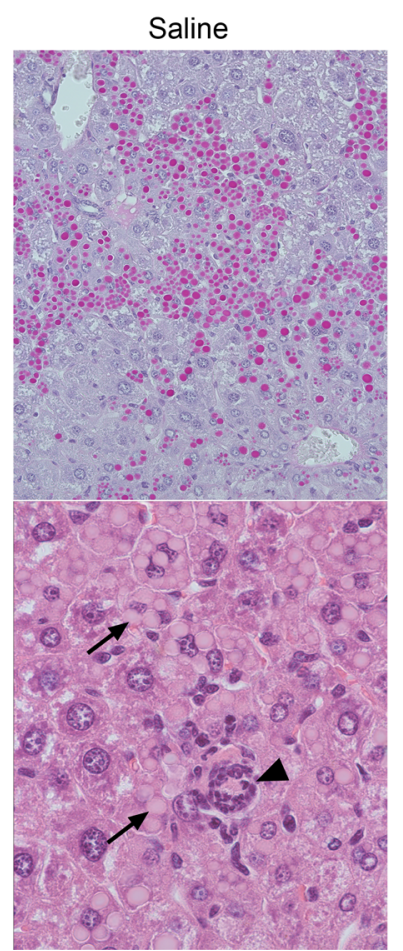
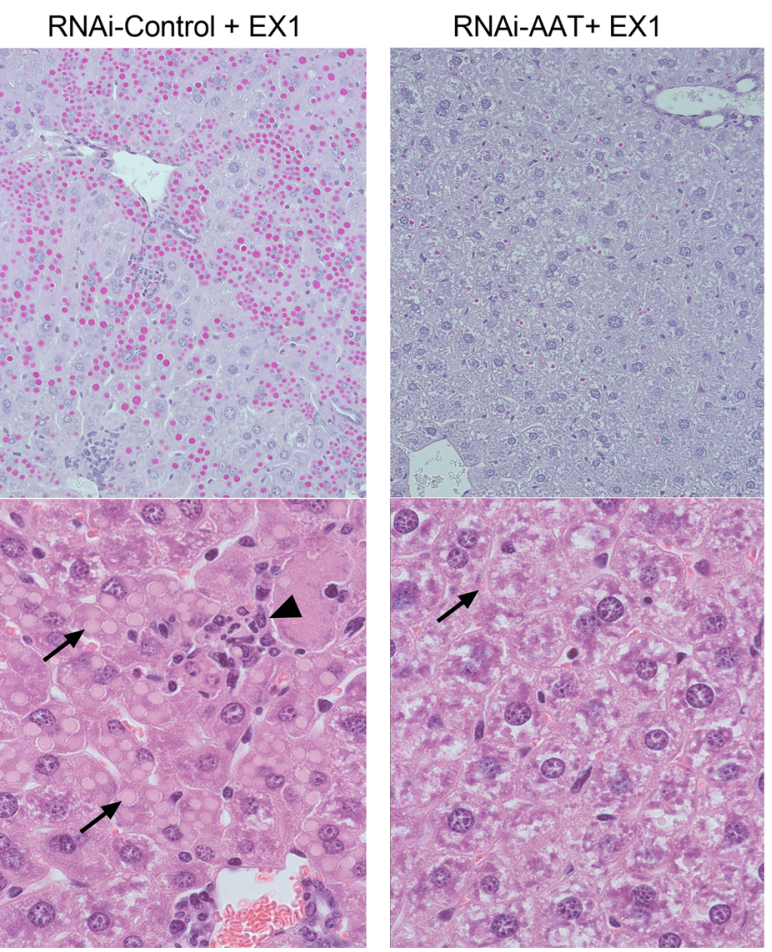

Figure 1. RNAi-mediated reduction of Z-AAT protein and mRNA in young PiZ mouse livers prevented polymer accumulation. Five-week-old male and female PiZ mice were given IV injections of $8 \mathrm{mg} / \mathrm{kg}$ ARC-AAT API plus $8 \mathrm{mg} / \mathrm{kg}$ EX1 (RNAi-AAT + EX1) once every 2 weeks (Q2W, $n=24$ ) or once every 3 weeks (Q3W, $n=8$ ) or were given Q2W injections of RNAi-Control plus $8 \mathrm{mg} / \mathrm{kg}$ EX1 (RNAi-Control + EX1) $(n=6)$ ). Age-matched control animals were injected Q2W with saline $(n=13)$. Untreated baseline mice were euthanized at 5 weeks of age $(n=5)$. (A) Plasma was collected at the indicated times and Z-AAT protein measured, shown as the group mean \pm SEM relative to pretreatment expression. (B) RNAi-AAT + EX1, RNAi-Control + EX1, and saline-injected mice were euthanized 2 weeks after final injection, and amounts of Z-AAT mRNA in homogenized liver tissue were quantified relative to the geometric mean of the saline group. Males were given 4 injections and euthanized when 13 weeks old. Females were given 5 injections and euthanized when 15 weeks old. (C and D) 
The amounts of soluble (monomeric) and insoluble (polymeric) Z-AAT protein in liver lysates of male mice at baseline (5 weeks old) or given 4 Q2W injections and euthanized when they were 13 weeks old were measured by semiquantitative Western blotting, shown relative to the saline group as the mean \pm SEM, $n=3-10$. (E) Representative PAS-D-stained (upper row) and H\&E-stained (lower row) liver sections from male mice at baseline or injected Q2W with saline, RNAi-Control + EX1, or RNAi-AAT + EX1. Scale bars for PAS-D indicate $50 \mu \mathrm{m}$ and for H\&E indicate $20 \mu \mathrm{m}$. Arrows point to Z-AAT globules and arrowheads to foci of inflammatory cells.

32/33-week study, the number of these foci in the saline-injected PiZ mice was 20.0-fold greater, and the affected area of the liver was 12.2-fold greater than at baseline. In contrast, the mice treated with ARCAAT had about 24-fold fewer foci of inflammatory cells, and these covered about 23-fold less area of liver relative to the saline-injected group. Consistent with the relatively low amount of inflammation in AATD that results in slowly progressing disease, the overall area of inflammation in these saline group mice was only $0.8 \%$ of the liver specimens.

PiZ mice that were treated for 32 weeks with a suboptimal dose of ARC-AAT $(4 \mathrm{mg} / \mathrm{kg})$ demonstrated a more limited response to treatment (Supplemental Figure 7). Knockdown of plasma Z-AAT with 4 mg/ $\mathrm{kg}$ was similarly effective to that with $8 \mathrm{mg} / \mathrm{kg}$ ARC-AAT 1 week after dosing but had rebounded approximately $50 \%$ by 2 weeks (Supplemental Figure $4 \mathrm{~A}$ ). Measured every other week before redosing, plasma Z-AAT was reduced approximately 50\% with repeat dosing (Supplemental Figure 7A). Mice treated with the lower dose had $63 \%$ less monomer in the liver, relative to baseline, which was considerably less reduction than the mice treated with $8 \mathrm{mg} / \mathrm{kg}$ ARC-AAT that had 93\% reduction (Supplemental Figure $7 \mathrm{~B}$ ). The amount of monomeric Z-AAT in the liver was consistent with the amount in the plasma measured every 2 weeks just before redosing. Treatment with the suboptimal dose appeared to slightly reduce polymer, relative to baseline, but the study was insufficiently powered to determine significance (Supplemental Figure 7C). This suboptimal dose prevented the polymer accumulation that occurred in saline-injected controls.

Production of plasma Z-AAT protein in mice treated with $8 \mathrm{mg} / \mathrm{kg}$ ARC-AAT returned to the same level as in the saline group approximately 8 weeks after the last dose of ARC-AAT (Supplemental Figure $8 \mathrm{~A})$. Likewise, the amount of monomeric Z-AAT in the liver was the same in the saline group as in groups that had been treated 32 weeks with $8 \mathrm{mg} / \mathrm{kg}$ ARC-AAT but then were off treatment for 8 or 16 weeks (Supplemental Figure 8B). However, the amount of polymeric Z-AAT accumulated more slowly after the end of therapy (Supplemental Figure 8, C and D). Sixteen weeks after the end of ARC-AAT treatment, levels of polymer had not recovered to those of the saline group. Eight weeks after the end of treatment when polymer had not yet increased, the liver appeared histologically normal without accumulation of large globules or inflammatory foci (Supplemental Figure 8D).

$A R C$-AAT treatment prevented PiZ disease-related gene expression. AATD liver disease-associated genes are upregulated in PiZ mice (30). An investigation into the ability of RNAi treatment to prevent disease-associated gene expression was conducted in young adult male PiZ mice (11-17 weeks old) treated with ARC-AAT or saline for 16, 24, or 32/33 weeks (Supplemental Figure 9). For comparison with age-matched WT animals, expression of these genes in young adult (15 weeks old) and in aged WT mice (48-78 weeks old) was measured. Fibrogenic collagen and metalloproteinase genes (COL1A1, COL1A2, COL3A1, MMP2, $M M P 12, M M P 13, T I M P 1)$ in young adult mice were similarly expressed between the WT and PiZ mice, but over the following months expression of these genes increased in PiZ mice (Supplemental Figure 9, Table 1 , and data not shown). Production of Z-AAT polymer is known to upregulate expression of not only fibrosis-associated genes but also genes associated with cellular stress, autophagy and apoptosis (GPNMB, NUPR1, LGALS3), and redox regulation (CBR3, GSTM3). Melanoma-associated transmembrane glycoprotein GPNMB is involved in cell proliferation, linking macroautophagy to phagocytosis (31). Nuclear protein transcriptional regulator 1 (NUPR1) is a chromatin binding protein that converts stress signals into a program of gene expression. Galectin 3 (LGALS3) plays a role in apoptosis and inflammation. Carbonyl reductase (NADPH) 3 (CBR3) is an NADPH-dependent oxidoreductase shown to be upregulated under conditions of oxidative stress (32). GSTM3 is glutathione- $S$-transferase of the $\mu$ class that detoxifies products of oxidative stress. Expression of these genes was higher in age-matched saline control-treated PiZ mice versus PiZ mice treated for 32-33 weeks with ARC-AAT (Table 1), the latter having similar expression of these genes as the age-matched WT mice (Supplemental Figure 9). This beneficial effect of ARCAAT treatment was also observed in mice treated for 16 or 24 weeks. The amount of $\alpha$-smooth muscle actin expression was no higher in PiZ mice than in age-matched WT mice, consistent with our observation that these mice inconsistently produce fibrils of collagen and rarely develop true fibrosis (29). 
A

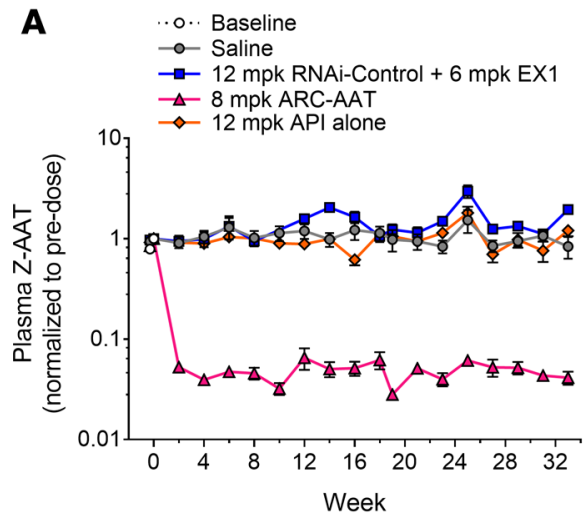

D

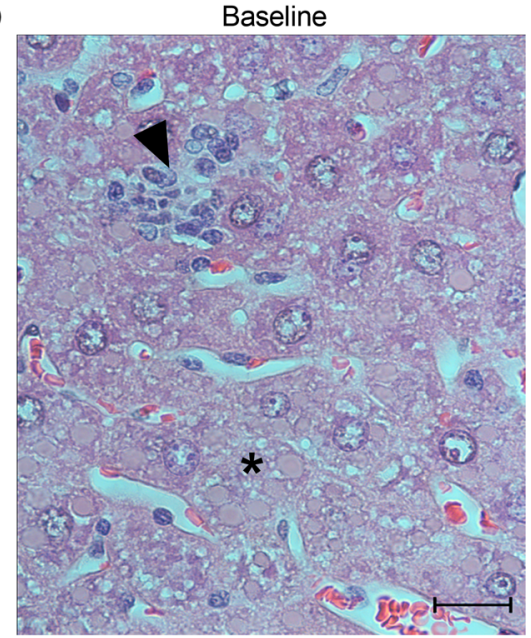

B

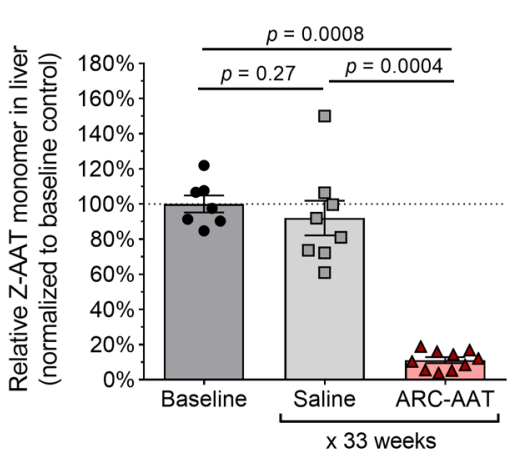

C

Z-AAT polymer

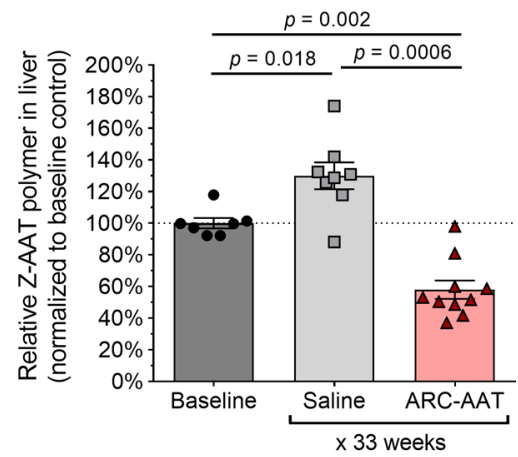

8 mpk ARC-AAT x 33 weeks
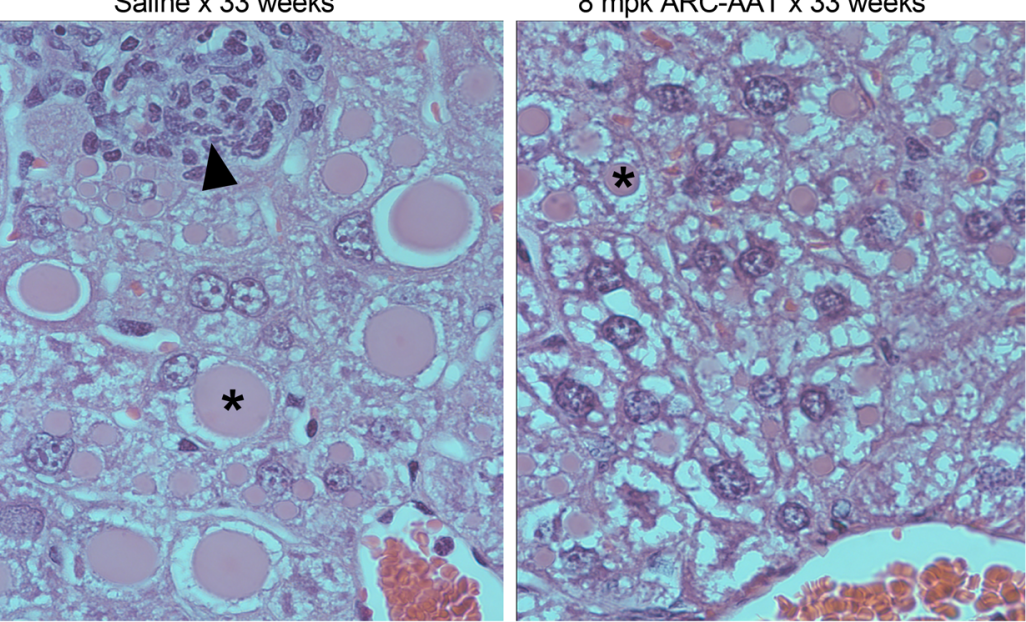
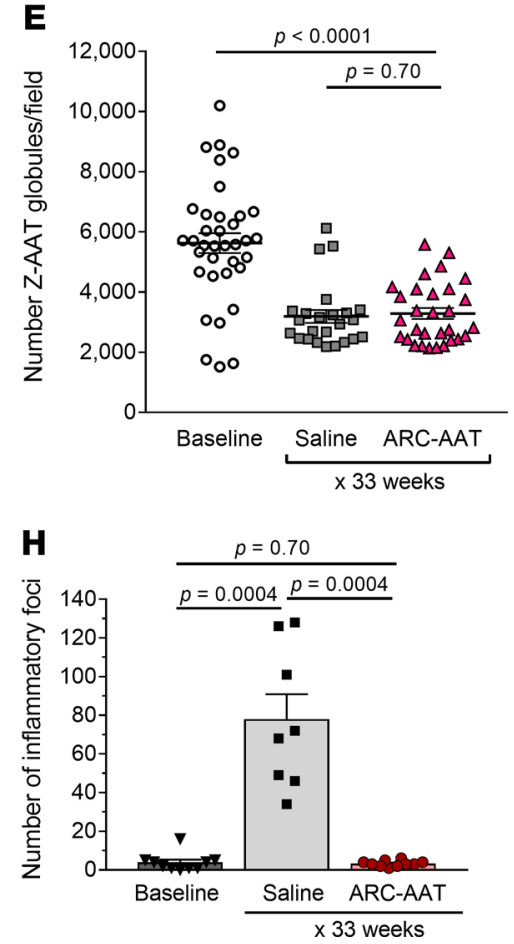

$\mathbf{F}$
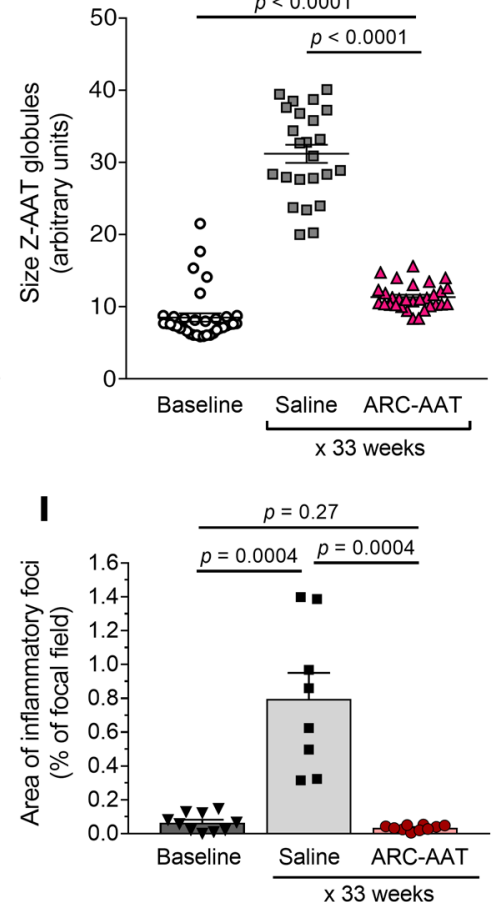

G

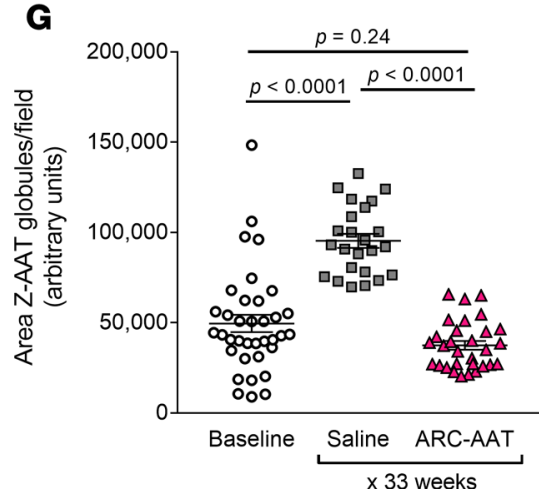

Figure 2. Sustained RNAi-mediated reduction of Z-AAT prevented liver disease and reversed polymer accumulation in adult PiZ mice. Male PiZ mice, 11-17 weeks old, were given IV injections Q2W of $8 \mathrm{mg} / \mathrm{kg}$ ARC-AAT ( $8 \mathrm{mg} / \mathrm{kg}$ ARC-AAT API + $4 \mathrm{mg} / \mathrm{kg} \mathrm{EX1),} 12 \mathrm{mg} / \mathrm{kg}$ RNAi-Control plus $6 \mathrm{mg} / \mathrm{kg}$ EX1, 12 $\mathrm{mg} / \mathrm{kg}$ ARC-AAT API alone, or saline $(n=4-12)$ for 30-31 weeks and were euthanized for evaluation 2 weeks after the final injection. Untreated baseline mice were euthanized at the start of the study. (A) Plasma was collected at the indicated times and Z-AAT protein measured, shown as the group mean relative to pretreatment. (B and $\mathbf{C}$ ) The amounts of monomeric and polymeric Z-AAT protein in liver lysates were measured by semiquantitative Western 
blotting, shown relative to baseline for individual animals and as the group means \pm SEM. (D) Representative H\&E liver sections from PiZ mice at baseline or following 33 weeks of saline (age-matched control) or ARC-AAT treatment. Asterisks indicate globules; arrowheads point to foci of inflammatory cells. Scale bar: $20 \mu \mathrm{m}$. (E-G) Z-AAT globules in PAS-D-stained liver sections were quantified in 3 fields of view for each animal ( $n=8-12$ ) for the number of globules/field of view (E), the size of globules (F), and the area of the specimens containing globules (G). (H and I) The number of inflammatory foci and area of liver specimens containing inflammatory foci are compared for baseline, saline-injected, and ARC-AAT-treated mice. Means are shown with SEM. Comparisons between groups (B, C, and E-I) were performed using nonparametric Wilcoxon's rank sum test.

Ultrastructural damage of PiZ liver reversed by ARC-AAT treatment. The ultrastructural effects of reducing Z-AAT protein in the liver were evaluated in a subset $(n=3)$ of mice from the prior study (Figure 2). Male PiZ mice, approximately 3 months old at baseline, were treated for 30-31 weeks with Q2W IV injections of ARC-AAT ( $8 \mathrm{mg} / \mathrm{kg}$ ) or saline and euthanized for EM evaluation at 32-33 weeks, 2 weeks after the final injection. At the end of the study, mice were approximately 11 months old, and the ultrastructure of their hepatocytes was compared with that of an age-matched WT mouse (Figure 3, Figure 4, Supplemental Figure 10, and Supplemental Figure 11).

The rERs of PiZ mice at baseline were dilated in comparison with those of the WT mouse (Figure 3). Segments of highly dilated cisternae formed multiple globules per cell. Globules enlarged over the course of 7 months in saline-injected PiZ mice. Between the large globules and the nucleus, hepatocytes were conspicuously devoid of the abundant rER that is observed around the nucleus in WT mouse hepatocytes. In contrast, the rERs in mice treated with ARC-AAT were abundant and much less dilated than in PiZ mice at baseline, instead resembling those of the WT mouse.

Autophagosomes are identified by an encompassing ribosome-free double membrane and internal contents that include debris, fragments of membranes, and membranous whirls (19). They pinch off from the ER and fuse with lysosomes (see Supplemental Figure 11, A, D, and E). Autophagosomes also encompass and digest damaged mitochondria. WT mouse hepatocytes contain autophagosomes (Figure 3B and Supplemental Figure 10), but those of PiZ mice at baseline and of the older saline group mice had more than the WT mouse, consistent with their role in degradation of Z-AAT polymer (Figure 3 and Supplemental Figure 11). Hepatocytes of mice treated with ARC-AAT had notably fewer autophagosomes and in this feature resembled the WT mouse (Supplemental Figure 11, M-R).

Retention of Z-AAT polymer in hepatocytes is known to result in oxidative stress, mitochondrial damage, and autophagy of damaged mitochondria (21). Healthy mitochondria have a more uniform appearance with some visible cristae (Figure 3, A and B; and Supplemental Figure 10) compared with the uneven staining of depolarized mitochondria, the dissolution of internal structures, and the condensates of multilamellar membranes seen in those that are damaged (Figure 3, D-F; and Supplemental Figure 11, D, E, and G-L). Mitochondria of PiZ mice at baseline were numerous, but many had the abnormal appearance of being in various stages of damage (Figure 3, Figure 4, and Supplemental Figure 11). The older saline group mice had a notable paucity of mitochondria with $14 \% \pm 5 \%$ of those that remained showing evidence of damage (Figure 3, E and F; Figure 4D; and Supplemental Figure 11, G-K). In contrast, mitochondria of mice treated with ARC-AAT were numerous and much more uniform in appearance than either baseline or saline group PiZ mice (Figure 3, G and $\mathrm{H}$; and Supplemental Figure 11, M-R), instead closely resembling those of the WT mouse. Only $2.7 \% \pm 2 \%$ of the mitochondria in mice treated with ARC-AAT were injured. Hepatocytes with globules exhibited distinctly more injury than globule-devoid cells. Unlike hepatocytes of PiZ mice at baseline or the older saline-injected animals, most mitochondria of ARC-AAT-treated PiZ mice appeared to be healthy, even in hepatocytes that contained globules (Supplemental Figure 11, Q and R).

Impaired mitochondrial function can lead to microvesicular steatosis, in which microvesicular fat is observed within hepatocytes (33). As previously reported (29), we also observed microvesicular steatosis in older (saline group) PiZ mice (Figure 4 and Supplemental Figure 11). A few fat droplets were observed in hepatocytes of mice treated with ARC-AAT, primarily those containing a globule, and in the age-matched WT mouse, but these were generally smaller and less frequent than in saline group mice (Supplemental Figure 10).

$R N A$-mediated reduction of Z-AAT protein reduced liver tumor formation in PiZ mice. PiZ mice develop adenomas and HCC with age. An assessment of the role ARC-AAT could play in tumor prevention was conducted in male PiZ mice that were 64-69 weeks (15/16 months) old at baseline. One group was given 16 Q2W IV injections of ARC-AAT $(8 \mathrm{mg} / \mathrm{kg})$, and a control group was injected with saline. Both groups were euthanized for histological evaluation after 32 weeks and compared with the 15/16-month-old mice euthanized at baseline $(n=8-9)$. 
Table 1. AAT RNAi treatment reduced expression of PiZ disease-associated genes

\begin{tabular}{|c|c|c|}
\hline \multicolumn{2}{|c|}{ Genes overexpressed in PiZ mice } & Fold reduction in ARC-AAT-treated PiZ mice relative to age-matched saline group \\
\hline COL1A1 & Collagen type I alpha 1 chain & 5.0 \\
\hline COL1A2 & Collagen type I alpha 2 chain & 3.9 \\
\hline COL3A1 & Collagen type III alpha 1 chain & 2.3 \\
\hline LGALS3 & Lectin, galactose binding, soluble 3 & 2.5 \\
\hline MMP2 & Matrix metallopeptidase 2 & 2.0 \\
\hline MMP12 & Matrix metallopeptidase 12 & 9.7 \\
\hline MMP13 & Matrix metallopeptidase 13 & 6.8 \\
\hline TIMP1 & Tissue inhibitor of metalloproteinase 1 & 19.7 \\
\hline NUPR1 & Nuclear protein transcription regulator 1 & 9.5 \\
\hline GPNMB & Glycoprotein (transmembrane) nmb & 12.0 \\
\hline CBR3 & Carbonyl reductase 3 & 10.1 \\
\hline GSTM3 & Glutathione-S-transferase, $\mu 3$ & 3.2 \\
\hline
\end{tabular}

The baseline PiZ mice in this study exhibited large globules (Figure 5, A-C). Unlike 3-month-old mice in which the globules were numerous, smaller, and panlobular (Supplemental Figure 12), globules in the 15/16-month-old mice were predominantly located pericentrally in Rappaport hepatic zones 2 and 3 (Figure 5A). Periportal areas were mostly globule free. Multiple small infiltrates of inflammatory cells $(99 \pm$ 16 foci per liver section), mostly neutrophils, surrounded necrotic hepatocytes that often contained a large globule (Figure 5B). Two of $8(25 \%)$ animals at baseline had liver growths of dysplastic or neoplastic cells. Most of the livers of baseline mice contained hepatocytes with giant hyperchromic nuclei and enlarged cytoplasm, features of compensatory hypertrophy that suggest transformative events had begun in these animals before tumors were apparent (Figure 5C).

At the end of the study, the saline control group mice were 23 months old. Their livers had fewer and mostly smaller globules compared with baseline, and these were primarily in the vicinity of the hepatic vein, zone 3 (Figure 5D). Livers of mice in this group displayed extensive parenchymal inflammation, sometimes accompanied by areas of necrosis (Figure 5, D-F). Globule accumulation and inflammation were associated with inflammatory foreign bodies in the livers of some saline group mice (Figure 5, F and $\mathrm{H})$. A regenerative parenchymal nodule, known to participate in hepatocarcinogenesis (34), was observed in 1 animal that also had a hepatic adenoma (Figure 5, H and I). Seven of $8(88 \%)$ animals had dysplastic or neoplastic growths that consisted of adenomas, well-differentiated HCC, enlarged aplastic hepatocytes, and complete loss of normal hepatic architecture (Figure 5, G, I-L, and P). In some animals the tumors occupied a substantial part of the liver volume (Figure 5, J and K). Inflammation was prevalent in livers with growths of neoplastic or dysplastic cells but not measured because extensive inflammation was not confined to foci and because much of the liver contained transformed cells.

Measurement of plasma and liver Z-AAT protein indicated these were reduced, relative to baseline, in the 23-month-old saline control group PiZ mice. Plasma Z-AAT protein was reduced by more than $80 \%$ in animal 318 that had large tumors and by an average of $41 \%$ in the group overall (data not shown). Western blotting showed reduced Z-AAT protein in the liver (Supplemental Figure 13). Consistent with previous reports that proliferating hepatocytes contain fewer globules, we measured a $70 \%$ reduction in polymeric Z-AAT $(P<0.0001)$ as well as a $38 \%$ reduction in monomeric Z-AAT $(P=0.037)$, relative to baseline.

Mice treated with ARC-AAT overall had a small number of hepatocytes containing globules and had minimal parenchymal inflammation (Figure $5, \mathrm{M}$ and $\mathrm{N}$ ). Inflammation was reduced to $24 \pm 8$ foci per liver section, a $76 \%$ reduction from baseline. Five of 9 had no evident dysplastic or neoplastic growths, and the liver exhibited a normal appearance, other than rare globules (Figure 5, G and P). Plasma Z-AAT was reduced approximately $80 \%-95 \%$ during the study in all but one of the ARC-AAT-treated animals, which is described below. The Z-AAT monomer was reduced by a mean of $74 \%(P=0.0002)$ in the livers of mice treated with ARC-AAT (Supplemental Figure 13), greater reduction than in the saline control group of this study but less than in younger mice that had been treated with ARC-AAT. Z-AAT polymer was reduced by a mean of $68 \%(P=0.001)$ in the ARC-AAT-treated group relative to baseline, corresponding to the same magnitude of reduction as in the saline group (Supplemental Figure 13). 


\section{A}
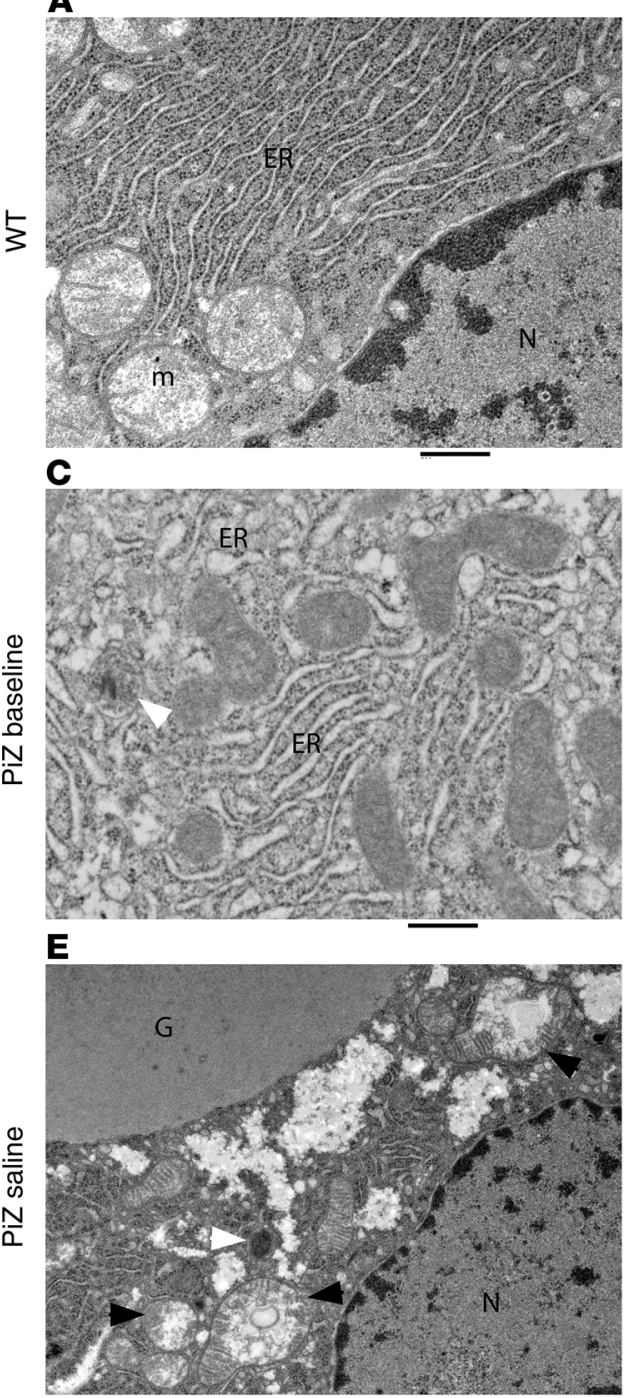

\section{G}

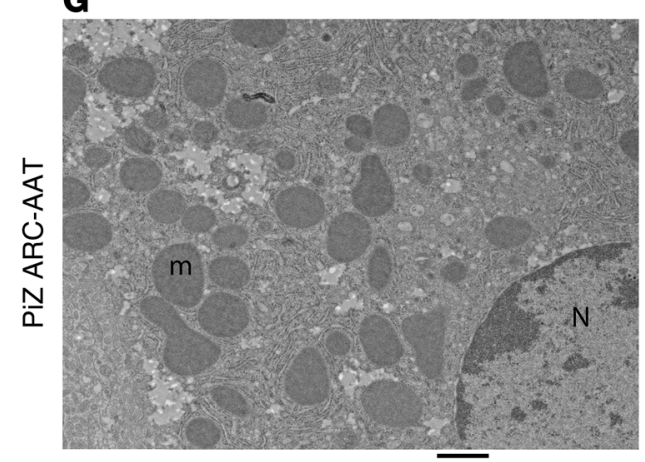

B

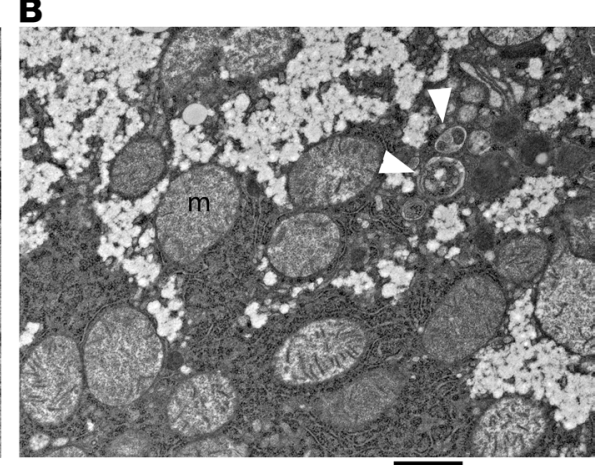

D

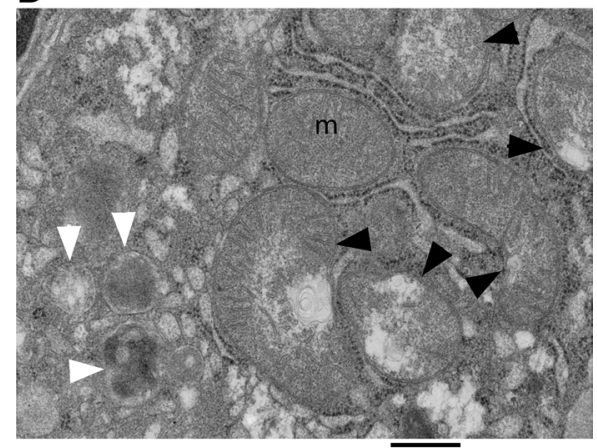

$\mathbf{F}$

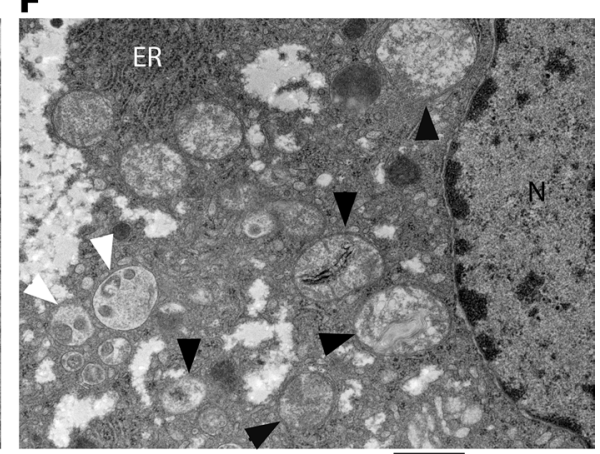

H

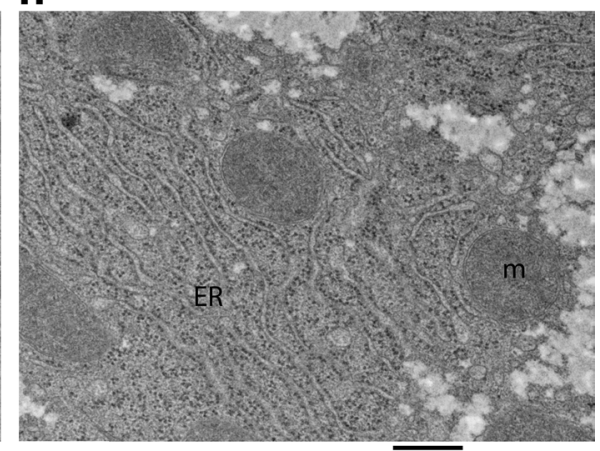

Figure 3. Sustained ARC-AAT treatment restored hepatocyte ultrastructure. EM images compare hepatocytes from a naive C57BL/6 WT mouse, 11 months old (A and B); PiZ mouse at baseline, 3 months old ( $\mathbf{C}$ and $\mathbf{D}$ ); PiZ mouse IV injected with saline (Q2W) for 33 weeks, 11 months old (E and F); and PiZ mouse IV injected (Q2W) with $8 \mathrm{mg} / \mathrm{kg}$ ARC-AAT for 33 weeks, 11 months old ( $\mathbf{G}$ and $\mathbf{H})$. G, globule; $\mathrm{N}$, nucleus; $\mathrm{m}$, healthy mitochondrion; ER, endoplasmic reticulum; black arrowheads, damaged mitochondria; white arrowheads, autophagosomes. Scale bars: $500 \mathrm{~nm}$ (A, C, D, and H), $1 \mu \mathrm{m}$ (B, E, and G), $800 \mathrm{~nm}$ (F).

Liver growths of dysplastic or neoplastic cells were observed in 4/9 (44\%) ARC-AAT-treated mice (Figure 5P). In 1 animal (number 291), most of the liver contained neoplastically transformed cells, adenomas, and HCC. In this animal, plasma Z-AAT protein was reduced $88 \%$ after the first ARC-AAT injection but was less reduced following subsequent injections, suggesting an alteration of hepatocytes early in the study. 
A
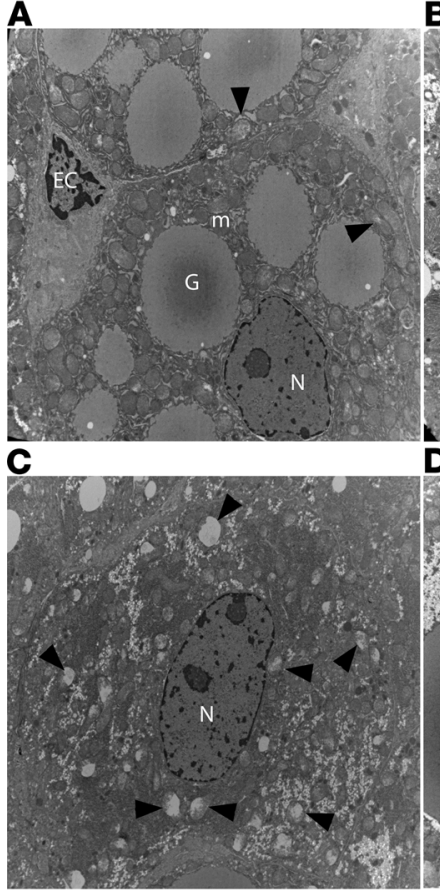

E

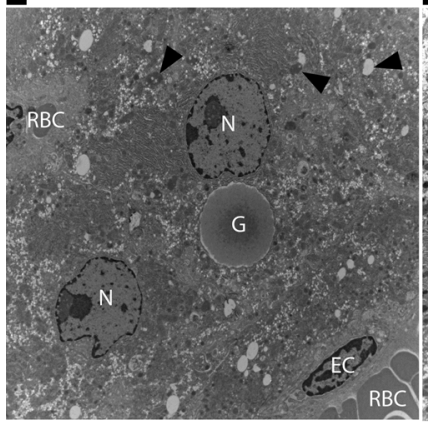

B

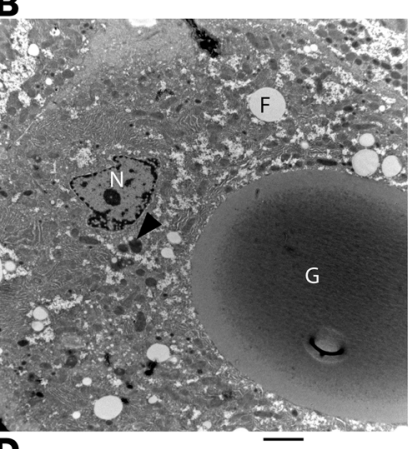

D

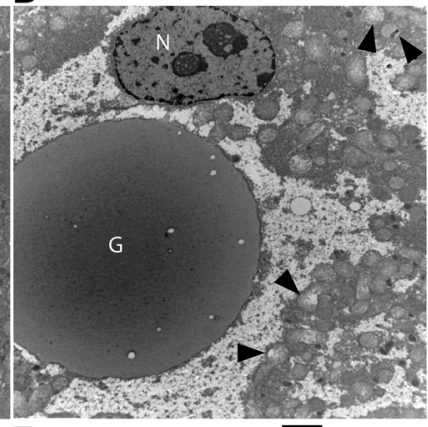

$\mathbf{F}$

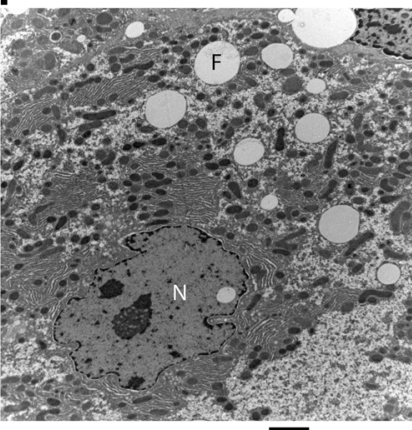

G

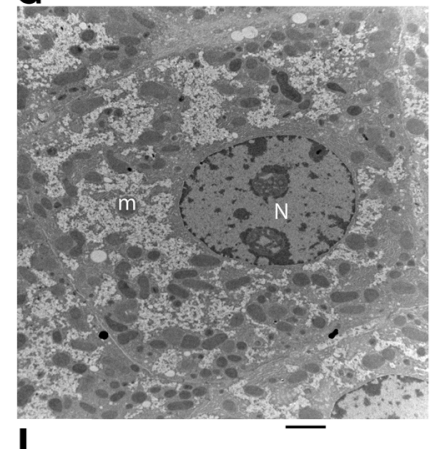

I
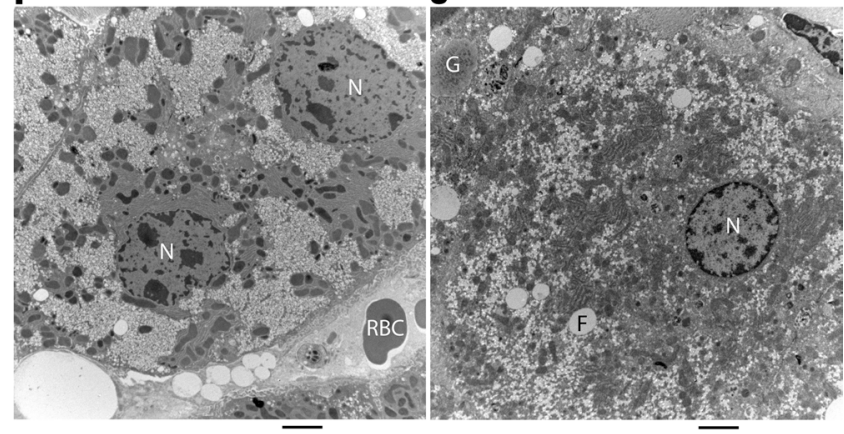

Figure 4. RNAi-mediated reduction of Z-AAT reversed ultrastructural damage to hepatocytes of PiZ mice. EM images from a PiZ mouse at baseline, 3 months old (A, C, and $\mathbf{E}$ ); a PiZ mouse IV injected with saline (Q2W) for 33 weeks, 11 months old (B, D, and F); a naive C57BL/6 WT mouse, 11 months old (C); and a PiZ mouse IV injected (Q2W) with 8 $\mathrm{mg} / \mathrm{kg}$ ARC-AAT for 33 weeks, 11 months old (H-J). G, globule; N, nucleus; $\mathrm{m}$, healthy mitochondrion; $\mathrm{F}$, microvesicular fat; RBC, red blood cell; EC, endothelial cell; black arrowheads, damaged mitochondria. Diameter of images in A, C, and $\mathbf{E}: 10 \mu \mathrm{m}$. Scale bar in other panels: $2 \mu \mathrm{m}$.

Three mice with smaller liver growths of dysplastic or neoplastic cells had a normal appearance of adjacent liver tissue (Figure $5 \mathrm{O}$ and Supplemental Figure 14). Animal 310 had dysplastic hepatocytes with significant globule content, although the liver architecture was not typical of adenomas or HCC (Supplemental Figure 14). Because ARC-AAT delivery requires uptake of both the cholesterol-conjugated RNAi trigger and the NAG-targeted EX1, the extensive globules suggest uptake of one or both compounds may have been altered in transformed hepatocytes. Transformations such as those observed in some baseline mice likely occurred before treatment of this group.

Hepatocytes containing large globules and previously described as "sick but not yet dead" are known to stimulate proliferation of globule-devoid cells $(11,14)$. The 15/16-month-old PiZ mice in this study had at baseline fewer globules than 3-month-old mice. Notably, globules were rare in the periportal regions (zone 1), where hepatocytes originate, but were observed in the vicinity of the hepatic veins, where the hepatocytes are typically older (zone 3). Globules and polymeric Z-AAT decreased substantially in the 2 groups of 23-monthold mice, those that were ARC-AAT treated and the saline group, and appear to have decreased to a similar degree relative to baseline. Remaining globules were near hepatic veins and appeared to be extruded into the veins (see supplementary materials and Supplemental Figure 12). Despite the similar loss of Z-AAT polymer between these 2 groups of old PiZ mice, the degree of tumorigenesis, inflammation, and necrosis differed distinctly, being lower in mice treated with ARC-AAT.

Subcutaneously administered RNAi therapeutic ARO-AAT. The goals for a new and improved RNAi therapeutic were to obtain potent and durable AAT KD from a subcutaneously administered RNAi trigger that had a robust therapeutic index. Development of this second-generation RNAi drug, named ARO-AAT, is described in the supplementary materials. A NAG targeting ligand for the asialoglycoprotein receptor 

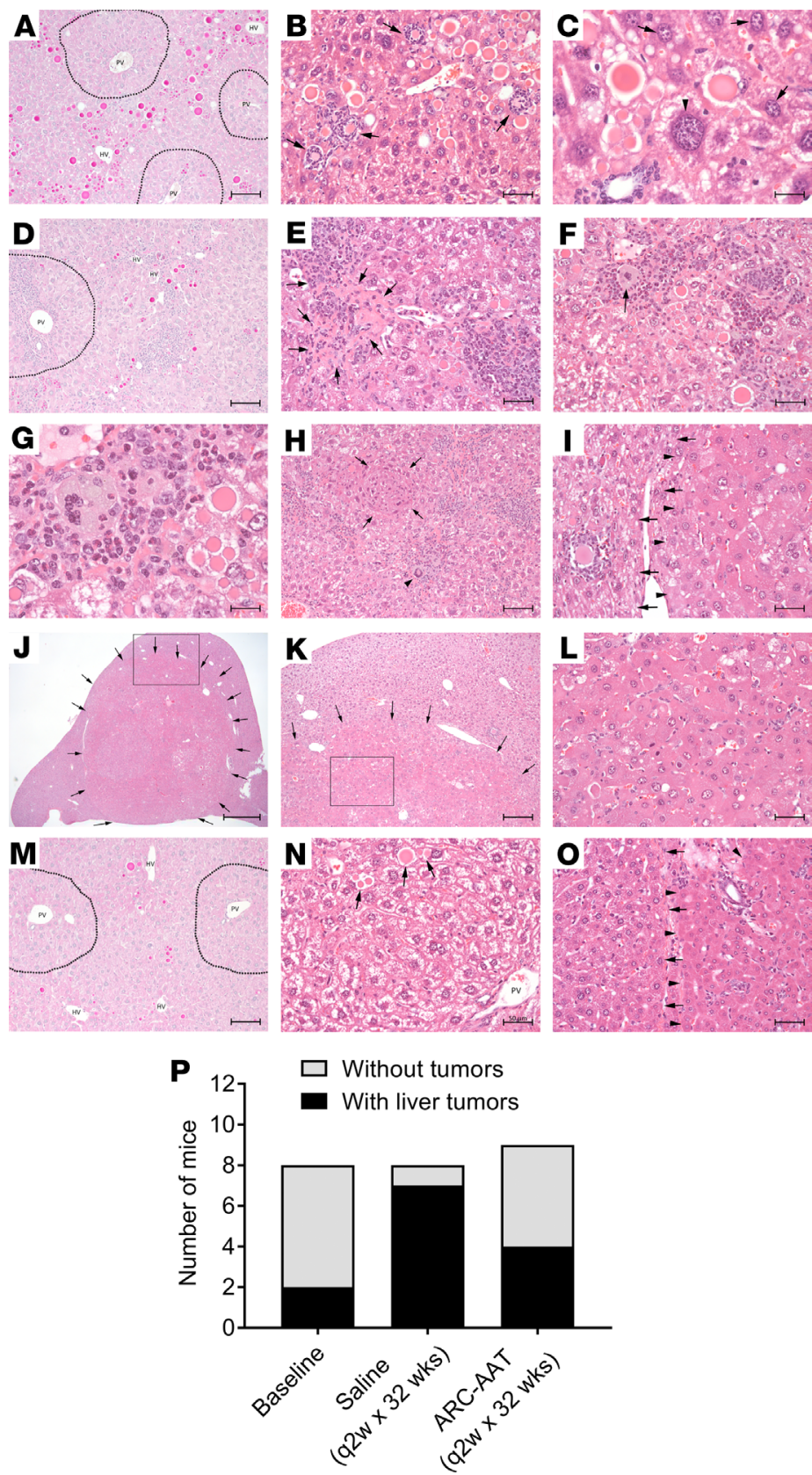

Figure 5. RNAi-mediated reduction of Z-AAT prevented tumor formation. Male PiZ mice 64-69 weeks old were euthanized at baseline (A-C) or given 16 Q2W injections of saline (D-L) or $8 \mathrm{mg} / \mathrm{kg}$ ARC-AAT (M-0) and euthanized at 32 weeks. (A, D, and M) PAS-D-stained liver, periportal areas indicated with dotted line; HV, hepatic vein; PV, portal vein; animal 277 in A, animal 309 in D, and animal 290 in $\mathbf{M}$. (B and C) $\mathrm{H} \& \mathrm{E}$-stained liver specimens from baseline animals. (B) Arrows indicate globules and cell debris surrounded by inflammatory cells in animal 277. (C) Arrowhead indicates enlarged hepatocyte with giant hyperchromic nucleus in animal 280. Arrows point to hepatocytes with normal-size nuclei. (E-L) H\&E-stained liver specimens from saline group mouse 309 showing globules, inflammation, and necrosis with inflammatory infiltration (arrows, E), inflammatory foreign body (arrow in $\mathbf{F}$ and $\mathbf{G}$, arrowhead in $\mathbf{H}$ ), and regenerative parenchymal nodule (arrows, $\mathbf{H}$ ). (I) Liver parenchyma-adenoma interface with arrowheads pointing to hepatic adenoma and arrows pointing to preserved liver parenchyma in animal 309. (J) Liver tumor occupies most of the left lateral lobe of saline group mouse 315 liver, indicated by arrows. (K) Arrows identify the tumor-parenchyma interface and inset shows hepatic adenoma with enlarged aplastic hepatocytes and complete loss of hepatic architecture in animal 315 (L). (N and $\mathbf{~ O ) ~ H \& E - s t a i n e d ~ l i v e r ~ s p e c i m e n s ~ f r o m ~ m i c e ~ t r e a t e d ~ w i t h ~}$ ARC-AAT. (N) Arrows point to rare globules in animal 282. (0) Liver parenchyma-adenoma interface with arrowheads pointing to hepatic adenoma and arrows pointing to preserved liver parenchyma in animal 279. (P) The numbers of animals with and without tumors are shown for the 8-9 animals in each group. Scale bars: $100 \mu \mathrm{m}(\mathbf{A}, \mathbf{D}, \mathbf{H}, \mathbf{I}, \mathbf{M}$, and $\mathbf{0})$ $50 \mu \mathrm{m}$ (B, E, F, L, and $\mathbf{N}$ ), $20 \mu \mathrm{m}$ (C and G), $1000 \mu \mathrm{m}$ (J), $200 \mu \mathrm{m}$ (K).

was attached to the sense strand of the RNAi trigger through a linker. The excipient EX1 was not used for delivery. Studies in PiZ mice demonstrated dose-dependent reductions of plasma Z-AAT protein and liver Z-AAT mRNA, as well as a dose exposure-response relationship between the mRNA reduction and the amount of RNAi trigger in liver tissue (Supplemental Figure 15).

$A R O-A A T$ deeply reduced $Z-A A T m R N A$ and protein in young PiZ mice. The efficacy of ARO-AAT (Figure 6) was evaluated in PiZ mice using the same study design as that for ARC-AAT treatment. PiZ mice were 5 weeks old at baseline before ARO-AAT treatment. One group of mice was given 4 Q2W SC injections of saline vehicle and euthanized at 13 weeks of age to observe age-dependent changes. Two groups were given 4 Q2W SC injections of $4 \mathrm{mg} / \mathrm{kg}$ RNAi trigger: either ARO-AAT or a NAG-targeted RNAi trigger modified similarly to ARO-AAT but targeting a nonmammalian gene as a negative control (RNAi-Control). These 2 groups were euthanized for evaluation 2 weeks after the final injection when they were 13 weeks old.

Each of the 4 ARO-AAT injections further reduced the plasma Z-AAT (Figure 6A), reflecting the durability of the response from prior injections. Z-AAT was reduced by a mean of $88.0 \%$ ( $0.9 \mathrm{log})$ following the first injection and $96.4 \%(1.4 \mathrm{log})$ following the fourth injection. Similar to plasma Z-AAT protein, the 
Z-AAT mRNA in the liver was reduced $94.5 \%(1.3 \mathrm{log})$ in ARO-AAT-treated mice, relative to baseline (Figure 6B). Both the plasma protein and liver mRNA increased over time in the control groups of PiZ mice.

At baseline, PiZ mice had 3 times as much polymeric as monomeric Z-AAT in the liver (data not shown), as measured by Western blotting, but the globules of polymer were small; the liver appeared histologically normal (Figure 6E). In the 13-week-old saline-injected or RNAi-Control-injected mice, polymeric Z-AAT in the liver was almost 6 times more than the monomeric Z-AAT (data not shown). Treatment of the mice with ARO-AAT reduced the amount of monomer in the livers by $97.7 \%(1.6 \mathrm{log})$ and the amount of polymer by $40.7 \%$, relative to baseline (Figure 6, C and D). In contrast, polymeric Z-AAT in livers from age-matched animals treated with saline or control RNAi trigger increased about $200 \%$ during the same period.

The number of globules in liver specimens from saline control mice sacrificed at 13 weeks old was 2.6fold greater than in the 5-week-old baseline mice (Figure 6F). The total area containing globules was 8.1fold higher and the average size of globules was 3.3-fold larger in saline-injected mice relative to baseline (Figure 6, G and H). Globule parameters in RNAi-Control-injected mice were statistically indistinguishable from those of saline group mice. Globules in ARO-AAT-treated mice were $74 \%$ reduced in total number, covered $89 \%$ less area of the liver, and were $63 \%$ smaller than those of the age-matched saline group mice. In summary, repeat dosing of ARO-AAT in young PiZ mice halted the normal progression of Z-AAT protein accumulation in the liver and allowed clearance of some preexisting polymer.

ARO-AAT mediates highly durable serum AAT reduction in human volunteers. Previously, human normal healthy volunteers (NHVs) were dosed with 0.38 to $8 \mathrm{mg} / \mathrm{kg}$ ARC-AAT in part A of single dose escalation study ARCAAT1001 (Supplemental Figure 16, reproduced with permission from Journal of Hepatology, ref. 25). Maximal AAT reduction occurred 4 weeks after dosing. A mean relative maximum reduction of $88.3 \%$ in serum AAT was observed at the $8 \mathrm{mg} / \mathrm{kg}$ dose level. Approximately $90 \%$ of endogenous AAT in humans and in NHPs is believed to be hepatocyte produced, based on the maximum endogenous AAT reductions we have observed (25). ARC-AAT was as effective at lowering serum AAT in patients with PiZZ in part B of the ARCAAT1001 clinical study as it was in the NHVs. The drug was well tolerated at efficacious doses, but the EX1 excipient was associated with NHP deaths at repeated high doses in a toxicology study (25). No toxicity was observed from the API alone, but the API did not reduce AAT in the absence of EX1 (Supplemental Figure 5).

In a phase I dose escalating clinical trial with ARO-AAT, NHVs were given a single subcutaneous dose of $35 \mathrm{mg}$ ARO-AAT or placebo in a blinded cohort (4 active: 4 placebo) or $100 \mathrm{mg}, 200 \mathrm{mg}$, or 300 mg ARO-AAT in open-label cohorts $(n=4)$ (Supplemental Figure 17). The wide margin of safety with a "no observed adverse event" level of $300 \mathrm{mg} / \mathrm{kg}$ in NHPs justified the ease of using a fixed dose level. A dose response was observed in the magnitude of serum AAT reduction as well as in duration (Figure 7A and Supplemental Figure 18). The lowest single dose of $35 \mathrm{mg}$ reduced serum AAT by $79.0 \% \pm 4.3 \%$ (SEM); the 100-mg single dose reduced serum AAT by $87.7 \% \pm 2.2 \%$; and the single doses 200 and 300 mg reduced it below the limit of quantitation with means of more than $91 \% \mathrm{KD}$. The nadir for all dose levels was attained approximately 6 weeks after dosing. The volunteers varied in body weight, so the fixed doses were also evaluated relative to body weight (Figure 7B), revealing a more precise dose response.

Serum AAT reductions were highly durable. AAT levels gradually began to increase from the nadir approximately 12 weeks after subjects were dosed with 35 or $100 \mathrm{mg}$ ARO-AAT, but remained well suppressed for at least 12 weeks in subjects dosed with 200 or $300 \mathrm{mg}$, and in these subjects increased only slightly by 16 weeks. All subjects were followed after the end of the study to demonstrate AAT return toward baseline. In all subjects for whom data were available, serum AAT increased from nadir at variable rates of return toward baseline.

This phase I trial in human subjects included a blinded, placebo-controlled, multidose arm. The NHVs were enrolled into 3 escalating dose cohorts (4 active: 4 placebo) to receive 100, 200, or $300 \mathrm{mg}$ of AROAAT or placebo on days 1, 29, and 57 (Supplemental Figure 19). Three months after the last dose, serum AAT was below the limit of detection in nearly all subjects.

ARO-AAT was well tolerated at doses up to $300 \mathrm{mg}$ given up to 3 times (Q4W). Most adverse events (AEs) were considered mild (Supplemental Table 3). There were no deaths and no serious or severe AEs reported. The most common AEs were upper respiratory tract infection (39\%) and headache (32\%). There was no statistically significant adverse difference in $\mathrm{FEV}_{1}$ changes from baseline between subjects receiving placebo and those receiving ARO-AAT.

In summary, ARO-AAT administered to human volunteers produced robust reductions in serum AAT levels from single or multiple doses. An average 90\% reduction in serum AAT was sustained for 6 weeks in 
A
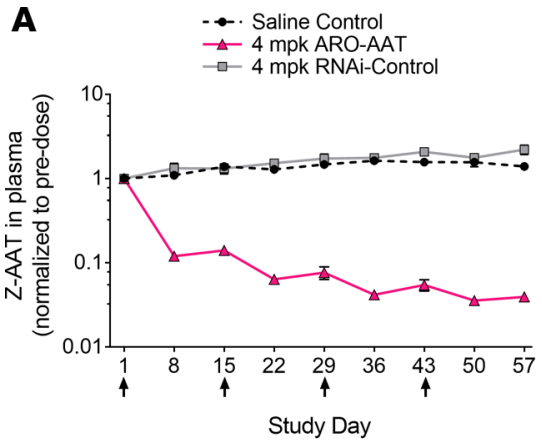

C

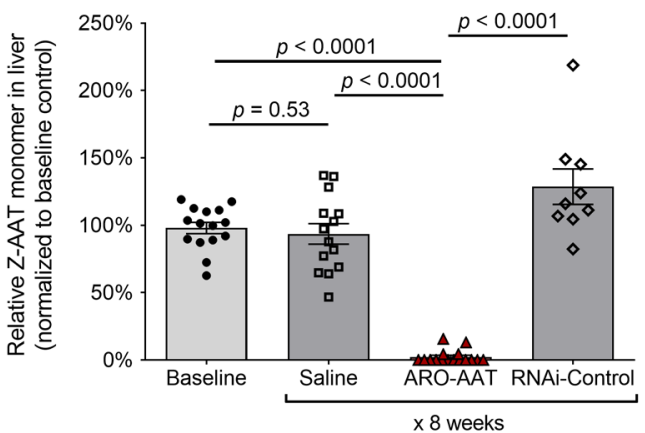

B

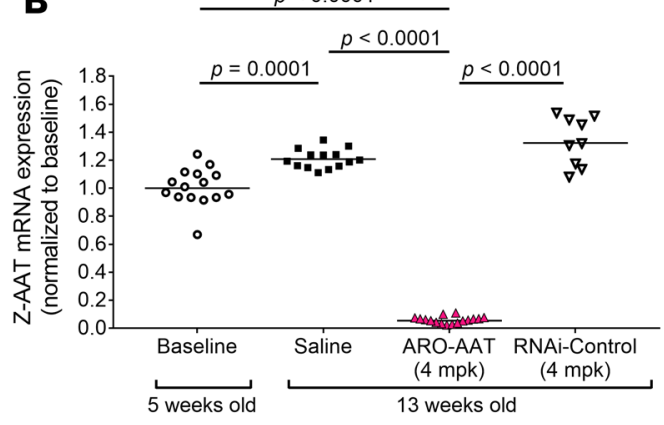

D

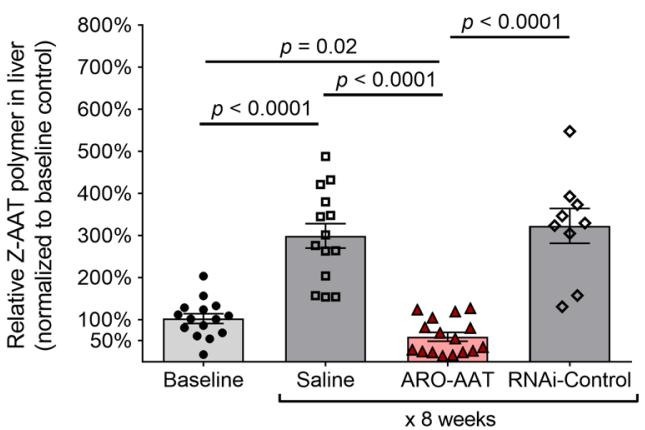

E

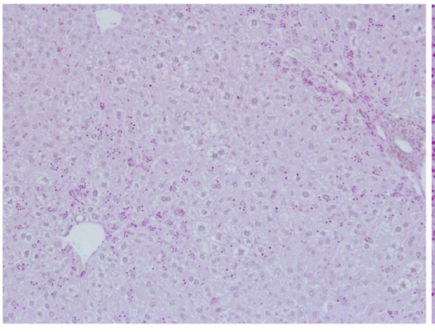

Saline

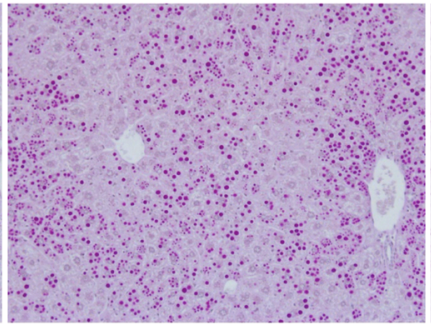

RNAi-Control
ARO-AAT

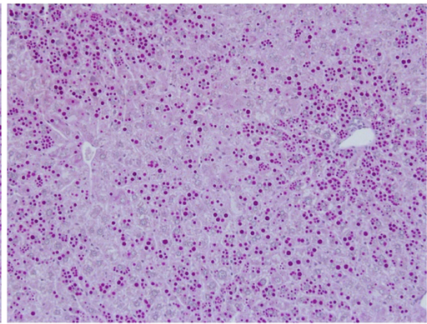

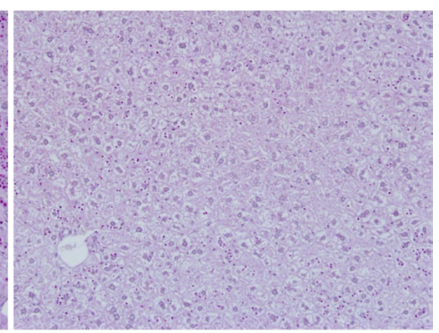

H

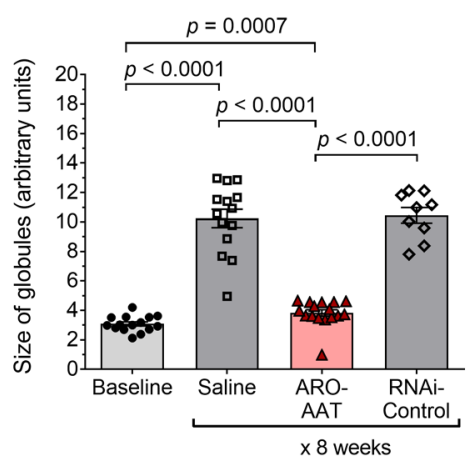

Size

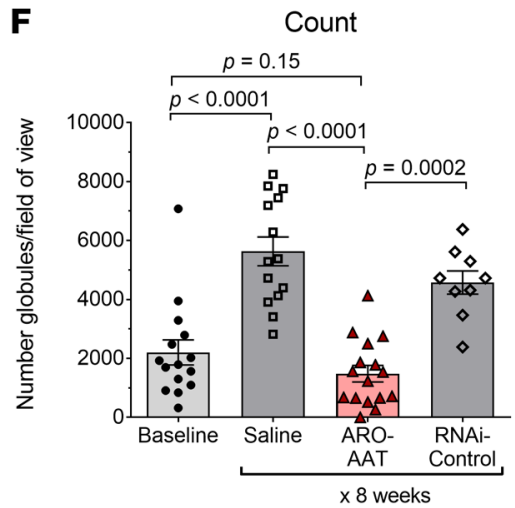

G

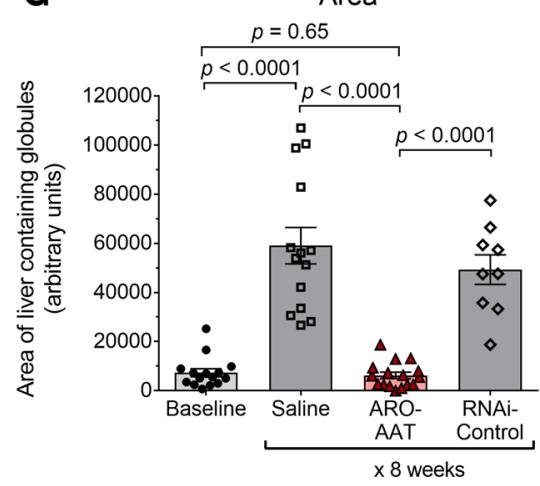

Figure 6. ARO-AAT treatment reduced Z-AAT protein and mRNA in young PiZ mice. Five-week-old male PiZ mice given 4 subcutaneous injections (Q2W) of $4 \mathrm{mg} / \mathrm{kg}$ ARO-AAT, saline, or RNAi-Control and were euthanized 2 weeks after the final injection $(n=9-16)$. Untreated baseline mice euthanized at 5 weeks of age $(n=15)$. (A) Plasma was collected at the indicated times and Z-AAT protein measured, shown as the group mean \pm SEM relative to pretreatment. (B) Amounts of Z-AAT mRNA in homogenized liver tissue were quantified relative to the geometric mean of the baseline group. (C and $\mathbf{D})$ The amounts of monomeric and polymeric Z-AAT protein in liver lysates of mice at baseline ( 5 weeks old) or after receiving 4 Q2W injections and then euthanized ( 13 weeks old) were measured by semiquantitative Western blotting, shown relative to the baseline group as the mean \pm SEM. (E) Representative PAS-D-stained liver sections from mice at baseline or injected Q2W with saline, RNAi-Control, or ARO-AAT. Globule number, globule size, and the area of the liver containing globules were measured by a densitometric method $(\mathbf{F}, \mathbf{G}$, and $\mathbf{H})$. Comparisons between groups in panels $\mathbf{B}-\mathbf{D}$ and $\mathbf{F}-\mathbf{H}$ were performed using nonparametric Wilcoxon's rank sum test. 

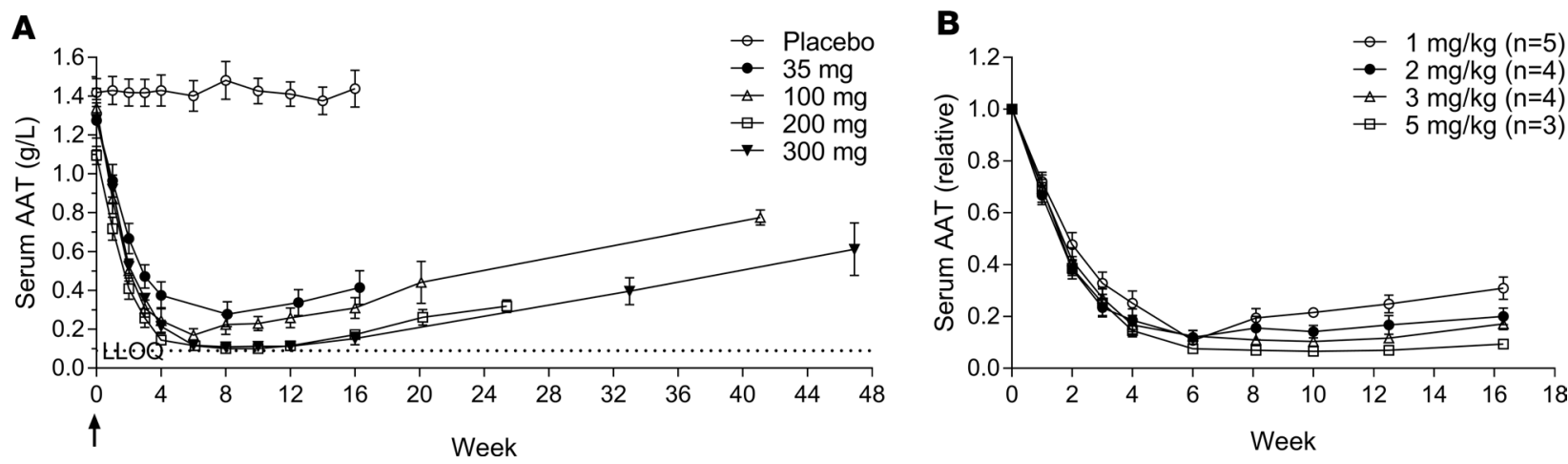

Figure 7. Serum AAT reductions in healthy human volunteers treated with ARO-AAT. Human subjects in a phase I dose escalating study were given a single SC injection of a fixed dose of $35 \mathrm{mg}, 100 \mathrm{mg}, 200 \mathrm{mg}$, or $300 \mathrm{mg}$ ARO-AAT or placebo $(n=4)$. (A) Serum was collected and AAT measured at the indicated times, shown as the group mean \pm SEM. (B) The doses were then divided by the subjects' body weight to assess the equivalent mg/kg doses. The lower limit of quantitation was $0.09 \mathrm{~g} / \mathrm{L}$ AAT.

200-mg and 300-mg single-dose cohorts. An average of greater than $90 \%$ reduction was sustained for more than 14 weeks in the 3-dose cohorts with 200 or $300 \mathrm{mg}$. This long duration of response supports the feasibility of quarterly dosing in patients with AATD. A potentially pivotal adaptive design phase II/III clinical trial is currently underway enrolling AATD patients with established hepatic fibrosis.

\section{Discussion}

RNAi as a treatment modality for AATD is intended to stop the production of Z-AAT protein in the liver, thereby preventing further accumulation of the disease-causing polymer and enabling the liver processes to gradually clear the preexisting polymer. Our studies in PiZ mice demonstrated that RNAi targeting AAT mRNA prevented the accumulation of nascent polymer and reduced preexisting polymer. The process of eliminating the globules was relatively slow, as some remained months after continuous and significant Z-AAT KD. Abnormal ER in PiZ hepatocytes manifests not only as inclusion bodies (globules) sequestered from the rest of the ER but also as dilated ER throughout hepatocytes. The formation of globules has been described as a mechanism to sequester toxic polymer and thereby protect cells, although some authorities consider it a sign of cell stress (19). By ultrastructural analysis we observed that despite the continued presence of some globules in hepatocytes, RNAi treatment mediated numerous changes in the liver that ameliorated the disease phenotypes. The most striking changes were normalization of rER throughout the hepatocytes and the appearance of abundant mitochondria resembling those in WT mice.

Retention of Z-AAT protein leads to ongoing mitochondrial injury (21). Damaged mitochondria in PiZ livers have been observed in various stages of injury before autophagocytosis. Oxidative stress coincident with the mitochondrial injury contributes to liver damage (30). The nearly normal ultrastructural appearance of RNAi-treated PiZ mouse hepatocytes was a stark contrast to the hepatocytes of mice that had not received RNAi treatment. Mitochondria in the latter mouse livers were scarce and predominantly injured. Damaged mitochondria are a source of reactive oxygen species that in turn react with macromolecules such as polyunsaturated lipids, resulting in oxidation of plasma and mitochondrial membranes, causing further liver injury. Mitochondrial dysfunction is associated with microvesicular steatosis, the consequence of a severe impairment in $\beta$-oxidation of fatty acids, and is a feature previously observed in older PiZ mice $(29,33)$. Accumulating evidence indicates a direct link between mitochondria, oxidative stress, and cell death, which may eventually lead to HCC (35). We also observed microvesicular steatosis in older untreated PiZ mice and further noted that it was associated with animals that had a paucity of healthy mitochondria and upregulated expression of genes that respond to oxidative stress. Steatosis has long been associated with AATD liver pathology $(36,37)$, and a recent study of PiZZ patients with undiagnosed liver disease noted an overrepresentation of steatosis in this group relative to the general population (8). RNAi treatment of PiZ mice resulted in predominantly healthy mitochondria and reduced microvesicular steatosis. Consistent with the restoration of healthy mitochondria as observed by EM, RNAi-treated mice had reduced expression of genes that respond to cellular and oxidative stress and those that promote autophagy and apoptosis. 
Globules in 1-year-old male PiZ mice were large, occupying much of the cytoplasmic space. Prior studies in PiZ mice revealed that hepatocytes with the highest burden of Z-AAT accumulation were most susceptible to injury and death (10). Globule burden was shown recently to be associated with risk of liver injury and fibrosis in patients with PiZZ (8). Although these observations may seem contrary to the hypothesis that sequestration of the Z-AAT polymer in globules is a protective mechanism, injured mitochondria and the lack of metabolic space within the cell are potential confounding features that increase along with globule size. A partial hepatectomy study previously demonstrated reduced survival of PiZ relative to WT mice, suggesting decreased hepatic functional reserve (14).

Inflammation and expression of fibrosis-associated genes increased with age in PiZ mice (30). Fibrosis typically follows inflammation in the human liver. In a recent study of individuals with AATD who had not previously been diagnosed with liver disease, $35.1 \%$ were found to have clinically significant fibrosis $(F \geq$ 2) (8). Fibrosis-associated collagen and tissue remodeling gene expression were significantly upregulated in older PiZ mice, even though these mice rarely developed fibrosis (29). Studies in PiZ mice have historically shown development of collagen fibrils that stain with Sirius red, but in our studies, the control mice infrequently and inconsistently produced collagen fibrils; therefore, we did not pursue this analysis. However, we measured expression of multiple fibrosis-associated genes over the course of approximately 1 year. PiZ mice that were 3 months old expressed these genes at a similar level to WT mice. Over the course of the next several months, expression increased significantly. This upregulation of fibrosis-associated genes and inflammation were abrogated by RNAi treatment, which instead reduced expression levels to those of WT mice. A recent investigation into the epigenetic modifications of patients with AATD revealed that altered methylation patterns for genes involved in liver cancer, cell cycle, fibrosis, and immune function were present before clinically detectable liver disease (38). This methylation pattern was associated with globule burden. Taken together, these results suggest there may be value in measuring liver disease-associated gene expression, inflammation, and Z-AAT polymer burden, then potentially RNAi-mediated changes in these parameters.

In AATD, hepatocytes overloaded with globules have been described as "sick but not yet dead." These cells stimulate proliferation of globule-devoid hepatic progenitor cells $(11,14)$. This chronic regenerative stimulation leads to hepatocyte dysplasia, adenomatosis, and HCC. As has been previously reported, we also observed that as PiZ mice aged, the number of globules was reduced $(28,29)$. This was observed in RNAi-treated mice and age-matched controls, but the liver morphology of these groups was different. The size of the globules and the area of the liver containing globules were greatly increased in 1-year-old mice, relative to 3-month-old animals, but the older mice had fewer globules. The globules in the RNAi-treated mice of this age were smaller than their untreated counterparts, but both groups had a similar number of globules. The similar decrease in globule number in both groups is consistent with previous reports that decreasing globule numbers is a feature of PiZ mouse aging, resulting from proliferation of hepatocytes without globules. Whether this is observed in human disease remains unreported, but increased hepatic progenitor cells have been measured in human PiZZ livers in early stages of liver disease (11). Globule number and Z-AAT polymer amounts decreased further and to a similar degree in the approximately 2-year-old RNAi-treated PiZ mice and their age-matched controls. In contrast to the similar polymer reduction in these very old mice, liver morphology was strikingly different in RNAi-treated animals compared with age-matched controls. RNAi-treated mice had fewer and smaller growths of transformed cells (e.g., HCC). The parenchyma of the liver in RNAi-treated mice appeared histologically nearly normal even in mice with small tumors. All but one animal in the untreated group had extensive numbers of transformed hepatocytes and most had significant inflammation. New light has been shed recently on the role of inflammation in predisposing cells to the development of cancer and in promoting cancers (39).

Hepatic progenitor (oval) cells originate near the portal region, and apoptosis of hepatocytes preferentially occurs near the hepatic vein in zone 3 of the liver (40). Indeed, we observed globule-loaded hepatocytes in the vicinity of the hepatic vein that were undergoing apoptosis. Brunt et al. demonstrated that the liver was replenished with hepatic progenitor cells throughout the lives of PiZ mice (11). We noted that in older PiZ mice the hepatocytes near the portal vein rarely had globules, while some presumably older hepatocytes near the hepatic vein had large globules. Newly formed hepatocytes, such as those in young mice, do not initially contain globules, which at least partially explains how increasing the rate of hepatocyte proliferation could result in a reduction of globules. In addition, we observed hepatocytes that appeared to produce no Z-AAT protein (see supplemental materials). Hepatocytes without globules have been thought to have alterations in Z-AAT protein metabolism. 
Hepatocytes loaded with globules undergoing apoptosis drop out into the hepatic vein and release globules into the circulation. Cell-free globules from hepatocytes that had already died in the livers of old PiZ mice were also released into the hepatic vein. We speculate that these globules could be a major source of the Z-AAT polymer that has been detected in the blood and lungs of patients with AATD. Polymeric Z-AAT is thought to exacerbate AATD lung disease (41). These observations suggest the intriguing possibility that treatment of AATD liver disease with RNAi could paradoxically reduce lung disease progression, especially in conjunction with augmentation therapy to provide the lung-protective WT AAT. AATD-associated lung disease takes decades to develop and may not develop at all in PiZZ patients with extremely low Z-AAT levels. Additionally, RNAi reduction of liver-produced Z-AAT would not reduce extrahepatic Z-AAT production.

Overall, these results in PiZ mice treated with RNAi to deeply reduce human Z-AAT production in the liver point to the potential for RNAi to effectively treat liver disease in children and adults with PiZZ AATD, although the duration of treatment necessary or even the possibility to improve clinical outcomes is unknown.

Several of the initial studies in this report were conducted with ARC-AAT, but the activity of AROAAT compared favorably with that of ARC-AAT in PiZ mice and in human NHVs. ARO-AAT activity was unexpectedly more durable than that of ARC-AAT and, therefore, supports quarterly or even less frequent dosing of patients to maintain nearly complete $\mathrm{KD}$ of Z-AAT protein production in the liver. ARO-AAT is currently in a pivotal clinical trial designed to assess changes in liver histology and AAT monomer and polymer content on liver biopsy after chronic dosing (ClinicalTrials.gov NCT03945292), and it is hoped that this trial can provide important validation that the benefits of RNAi shown in PiZ mice in our studies can translate to PiZZ patients.

\section{Methods}

RNAi agents. RNAi trigger sequences and modifications are shown in Supplemental Table 1. Synthesis of RNAi triggers used standard phosphoramidite chemistry. Synthesis of EX1 was previously described (42).

Mouse procedures. PiZ mice maintained on a C57BL/6 background (strain Z11.03, ref. 13) were obtained from colonies maintained at Saint Louis University Doisy Research Center. Throughout the studies, animals had access ad libitum to water and chow. Each animal was dosed with $200 \mu \mathrm{L}$ dosing solution per 20 g body weight. Dosing solutions in studies containing ARC-AAT components were IV injected into the tail vein. Lyophilized EX1 was gently resuspended with sterile water for injection, then mixed with chol-RNAi (ARC-AAT API or RNAi-Control). Dosing solutions in studies containing ARO-AAT were SC injected into the nape of the neck. Blood was collected into heparinized capillary tubes, with the plasma isolated and stored at $-80^{\circ} \mathrm{C}$ until the time of assay. Mice were euthanized by carbon dioxide asphyxiation followed by cervical dislocation. Liver pieces for protein, mRNA, or RNAi trigger analysis were snap-frozen in liquid nitrogen and then stored at $-80^{\circ} \mathrm{C}$ until further processed. Additional liver pieces were fixed in $10 \%$ buffered formalin and then embedded in paraffin for histological evaluation of both H\&E and PAS-D with or without hematoxylin. Liver tissue for evaluation by EM was placed in $2.5 \%$ glutaraldehyde in $0.1 \mathrm{M}$ sodium cacodylate buffer $\mathrm{pH} 7.4$ and stored at $4^{\circ} \mathrm{C}$ until processing as previously described (19).

Human AAT ELISA. Quantitative measurement of human Z-AAT in mouse plasma was carried out using an in vitro competitive ELISA kit (catalog ab108798, Abcam, Cambridge, Massachusetts, USA). Plasma samples were diluted 100- to 400-fold in 1X Diluent N (Abcam) and assayed according to the manufacturer's instructions. Standards were included in the kit. Plasma Z-AAT concentration in each animal at a given time point was normalized to the predose amount.

$R N A$ analysis. Each frozen liver specimen was added to $4 \mathrm{~mL}$ of TRI Reagent RT (Molecular Research Center, Inc., Cincinnati, Ohio, USA) and homogenized using a Bio-Gen PRO200 tissue homogenizer (Pro Scientific, Inc., Oxford, Connecticut, USA) for approximately 30 seconds. Total RNA was isolated following the manufacturer's recommended protocol: $1 \mathrm{~mL}$ homogenate was added to $50 \mu \mathrm{L}$ 4-bromoanisole and mixed, and phases were separated by centrifugation. Then, $0.25 \mathrm{~mL}$ of aqueous phase was removed, precipitated with isopropyl alcohol, and centrifuged. The resultant pellet was washed with $75 \%$ ethanol and resuspended in $0.4-0.6 \mathrm{~mL}$ nuclease-free water. Total RNA was reverse-transcribed using the High Capacity cDNA Reverse Transcription Kit (Life Technologies, Thermo Fisher Scientific, Grand Island, New York, USA). The cDNA was diluted 1:50 and multiplex real-time quantitative PCR was performed with 4 replicates on a QuantStudio 7 Real-Time PCR System using TaqMan Gene Expression assays (both from Life Technologies, Thermo Fisher Scientific) as shown in Supplemental Table 2. Gene expression data were analyzed using the comparative CT method of relative quantification (43). 
Measurement of soluble and insoluble Z-AAT. Semiquantitation of monomeric and polymeric Z-AAT was performed as previously described $(26,27)$. Briefly, $10 \mathrm{mg}$ liver tissue was added to $2 \mathrm{~mL}$ lysate buffer at $4^{\circ} \mathrm{C}$ (50 mM Tris- $\mathrm{HCl} \mathrm{pH}$ 8.0, $150 \mathrm{mM} \mathrm{NaCl}, 5 \mathrm{mM} \mathrm{KCl}, 5 \mathrm{mM} \mathrm{MgCl}, 0.5 \%$ Triton X-100, and 1:100 Sigma Protease Inhibitor Cocktail (MilliporeSigma, St. Louis, Missouri, USA), homogenized in a prechilled Dounce homogenizer for 30 repetitions, then vortexed vigorously. Aliquots of $0.5 \mathrm{~mL}$ were passed through a 28-gauge needle 10 times. Protein concentration was determined by the Bicinchoninic Acid Kit method (Pierce BCA, Thermo Fisher Scientific, Waltham, Massachusetts, USA); then a 5- $\mu \mathrm{g}$ aliquot was centrifuged at 15,000 $g$ for 30 minutes at $4^{\circ} \mathrm{C}$. Supernatant was removed and placed in a separate tube. Supernatant (soluble fraction) and pellet (insoluble fraction) were separated by SDS-PAGE after denaturation of the insoluble polymers. Polymers were denatured and solubilized by addition of $10 \mu \mathrm{L}$ chilled cell lysis buffer ( $1 \%$ Triton X-100, 0.05\% deoxycholate, $10 \mathrm{mM}$ EDTA in phosphate-buffered saline), vortexed for 30 seconds, sonicated on ice for 10 minutes, and vortexed again. Then, 2.5X Sample Buffer (50\% 5X Sample Buffer [5\% SDS, 50\% glycerol, $0.5 \mathrm{M}$ Tris $\mathrm{pH}$ 6.8], $10 \% \beta$-mercaptoethanol, $40 \%$ double-distilled $\mathrm{H}_{2} \mathrm{O}$ ) was added to each soluble and insoluble sample at a volume of $50 \%$ of the sample volume. Samples were boiled and equal amounts (micrograms) of total liver protein loaded, separated into soluble/insoluble fractions. Following electrophoresis, proteins were transferred to nitrocellulose membranes. AAT was detected with primary antibody (goat anti-human alpha-1 antitrypsin, catalog 80502, discontinued, DiaSorin, Stillwater, Minnesota, USA) and secondary antibody (rabbit anti-goat IgG HRP conjugated, Dako North America, Inc., Carpinteria, California, USA) using the ECL 2 Western Blotting procedure (Thermo Fisher Scientific, Rockford, Illinois, USA) according to manufacturer recommendations. Densitometry analysis was carried out using ImageJ (NIH, Bethesda, Maryland, USA).

Histological analysis. Paraffin-embedded liver tissue was cut into $4-\mu \mathrm{m}$ sections. Sections were stained with PAS-D to visualize and quantify globule numbers, globule size, and total globule area using ImageJ. H\&E-stained specimens were evaluated by a pathologist blinded to sample identity. Morphometry (Sigma-Pro Imaging program, Systat Software Inc., Chicago, Illinois, USA) was performed to quantitate the area of inflammation. H\&E-stained slides were scanned and digitized using a photographic film scanner. Inflammation was determined by the pathologist viewing the slide under a microscope. The corresponding whole-slide scanned image was opened with SigmaScan Pro software and enlarged to the size of a computer screen for the areas of inflammation to be outlined. Total area of liver specimens and areas of inflammation were quantitated using this software to determine numbers of inflammatory foci and percentages of the specimen containing inflammation.

EM and microscopic features. Material for transmission electron microscopy was processed by standard techniques as previously described (19). Ultrastructural criteria for autophagy and injury were as previously described $(19,21,44)$. Briefly, the percentage of mitochondria with internal injury was quantified by counting individual mitochondria in 10 photomicrographs, each showing a randomly selected hepatocyte with its nucleus. Rigorous, specific ultrastructural criteria for injury were established before the analysis. Mitochondria were considered to have internal structural alterations if at least 1 accumulation of multilamellar membranes was visible within the outer limiting membrane of the mitochondrion itself and/or if the internal cristae and matrix were heterogeneously distributed within the outer limiting membrane of the mitochondrion, leaving open areas with membrane blebs in the organelle (10). Injured mitochondria were defined by the presence of at least 1 accumulation of multilamellar membranes within the limiting membrane of the mitochondrion and/or a change in the density of the internal structures of the mitochondrion. However, if density change alone was the only change in a given individual mitochondrion, then there had to be open areas with bleb formation in the outer membrane for it to be considered injured. Although diffuse, homogeneous density changes were seen in many mitochondria in the PiZ mice, this alteration was not by itself deemed sufficient for a designation as damaged.

Clinical trial of ARO-AAT in human volunteers. Normal healthy adult male and female volunteers (NHVs) in a single-center, randomized, double-blind, placebo-controlled single and multiple dose escalation study were 18-52 years old with BMIs between 19.0 and $35.0 \mathrm{~kg} / \mathrm{m}^{2}$ (clinical trial NCT03362242). NHV subjects enrolled sequentially into 7 cohorts. Cohorts 1 through 4 were randomized to receive ARO-AAT or placebo (4 active: 4 placebo) at a single dose of $35 \mathrm{mg}$ (cohort 1) followed by multiple escalating doses of $100 \mathrm{mg}$ (cohort 2), $200 \mathrm{mg}$ (cohort 3), and $300 \mathrm{mg}$ (cohort 4) administered as an SC injection. Cohorts 2b, 3b, and $4 \mathrm{~b}$ were open label consisting of 4 subjects receiving single doses of 100, 200, and $300 \mathrm{mg}$ ARO-AAT (Supplemental Figure 17 and Supplemental Methods). All subjects provided written informed consent.

Statistics. As appropriate for the study design, pairwise comparisons between baseline group and each treated group or between treated groups were performed using the nonparametric Wilcoxon rank 
sum test (SAS version 9.4). Comparisons for small group sizes in Supplemental Figure 7 were performed with GraphPad Prism version 7.03 for Windows (GraphPad Software) by pairwise Student's 2-tailed $t$ test. Significance was identified by $P<0.05$ ( 2 sided). $P$ values were reported without multiplicity adjustment.

Study approval. All mouse studies were conducted in accordance with the National Research Council's Guide for the Care and Use of Laboratory Animals (National Academies Press, 2011) and were approved by Saint Louis University IACUC (St. Louis, Missouri, USA) or the Arrowhead Pharmaceuticals IACUC (Madison, Wisconsin, USA). Clinical trial AROAAT1001 was approved by the Central Health and Disability Ethics Committee and New Zealand Medicines and Medical Devices Safety Authority (MedSafe/ SCOTT), Wellington, New Zealand. All subjects provided written informed consent.

\section{Author contributions}

CIW, RMP, SBK, DLL, and JHT designed and oversaw the PiZ mouse studies; CIW wrote the manuscript; KB performed mouse studies and acquired data; JHT conducted EM analysis; VMS evaluated histological parameters; JOH conducted NHP studies; CIW, KB, RMP, and DRC analyzed data and interpreted results; $\mathrm{JH}$ designed and managed the clinical study; BDG approved the design and monitored clinical results; CS, JK, and EG ran the clinical study; and QC, HLH, and MFBA analyzed samples.

\section{Acknowledgments}

This work was supported in part by the Alpha-1 Foundation. Special thanks to Lauren Almeida and Darren Wakefield for ARC-AAT development; Tao Pei, Rui Zhu, and Zhen Li for ARO-AAT development; Jenni Franey and Erin Touchette for assistance with mouse procedures; Riley Puntney for performing NHP procedures; Zhao Xu, Jacob Griffin, and Heather Sternard for RNAi trigger analysis; Aaron Anderson for technical assistance; Jason Klein, Collin Hagen, and Steven Knoll for material formulation; and Guofeng Zhang, Yinghua Bian, and Maria Afrazi for histology. Certain research in this publication was supported in part by the Office of the Director, NIH, under award P51OD011106 to the Wisconsin National Primate Research Center, University of Wisconsin-Madison. This research was conducted in part at a facility constructed with support from Research Facilities Improvement Program grant numbers RR15459-01 and RR020141-01. The content of this publication is solely the responsibility of the authors and does not necessarily represent the official views of the NIH.

Address correspondence to: Christine Wooddell, Arrowhead Pharmaceuticals, 502 S. Rosa Road, Madison, Wisconsin 53719, USA. Phone: 608.316.3930; Email: cwooddell@arrowheadpharma.com.

1. Greene CM, et al. $\alpha 1$-Antitrypsin deficiency. Nat Rev Dis Primers. 2016;2:16051.

2. Teckman JH, An JK, Loethen S, Perlmutter DH. Fasting in alpha1-antitrypsin deficient liver: constitutive [correction of consultative] activation of autophagy. Am J Physiol Gastrointest Liver Physiol. 2002;283(5):G1156-G1165.

3. Lomas DA, Evans DL, Finch JT, Carrell RW. The mechanism of Z alpha 1-antitrypsin accumulation in the liver. Nature. 1992;357(6379):605-607.

4. Carrell RW, Lomas DA. Alpha1-antitrypsin deficiency--a model for conformational diseases. N Engl J Med. 2002;346(1):45-53.

5. Chu AS, Chopra KB, Perlmutter DH. Is severe progressive liver disease caused by alpha-1-antitrypsin deficiency more common in children or adults? Liver Transpl. 2016;22(7):886-894.

6. Teckman JH, et al. Baseline analysis of a young $\alpha$-1-antitrypsin deficiency liver disease cohort reveals frequent portal hypertension. J Pediatr Gastroenterol Nutr. 2015;61(1):94-101.

7. Patel D, Teckman JH. Alpha-1-antitrypsin deficiency liver disease. Clin Liver Dis. 2018;22(4):643-655.

8. Clark VC, et al. Clinical and histologic features of adults with alpha-1 antitrypsin deficiency in a non-cirrhotic cohort. $J$ Hepatol. 2018;69(6):1357-1364.

9. Teckman JH, Mangalat N. Alpha-1 antitrypsin and liver disease: mechanisms of injury and novel interventions. Expert Rev Gastroenterol Hepatol. 2015;9(2):261-268.

10. Lindblad D, Blomenkamp K, Teckman J. Alpha-1-antitrypsin mutant Z protein content in individual hepatocytes correlates with cell death in a mouse model. Hepatology. 2007;46(4):1228-1235.

11. Brunt EM, Blomenkamp K, Ahmed M, Ali F, Marcus N, Teckman J. Hepatic progenitor cell proliferation and liver injury in $\alpha$-1-antitrypsin deficiency. J Pediatr Gastroenterol Nutr. 2010;51(5):626-630.

12. Sifers RN, Carlson JA, Clift SM, DeMayo FJ, Bullock DW, Woo SL. Tissue specific expression of the human alpha-1-antitrypsin gene in transgenic mice. Nucleic Acids Res. 1987;15(4):1459-1475.

13. Carlson JA, et al. Accumulation of PiZ alpha 1-antitrypsin causes liver damage in transgenic mice. J Clin Invest. 1989;83(4):1183-1190.

14. Rudnick DA, Liao Y, An JK, Muglia LJ, Perlmutter DH, Teckman JH. Analyses of hepatocellular proliferation in a mouse 
model of alpha-1-antitrypsin deficiency. Hepatology. 2004;39(4):1048-1055.

15. Rudnick DA, Shikapwashya O, Blomenkamp K, Teckman JH. Indomethacin increases liver damage in a murine model of liver injury from alpha-1-antitrypsin deficiency. Hepatology. 2006;44(4):976-982.

16. Geller SA, et al. Hepatocarcinogenesis is the sequel to hepatitis in Z\#2 alpha 1-antitrypsin transgenic mice: histopathological and DNA ploidy studies. Hepatology. 1994;19(2):389-397.

17. Marcus NY, et al. Characteristics of hepatocellular carcinoma in a murine model of alpha-1-antitrypsin deficiency. Hepatol Res. 2010;40(6):641-653.

18. Perlmutter DH. Autophagic disposal of the aggregation-prone protein that causes liver inflammation and carcinogenesis in alpha-1-antitrypsin deficiency. Cell Death Differ. 2009;16(1):39-45.

19. Teckman JH, Perlmutter DH. Retention of mutant alpha(1)-antitrypsin Z in endoplasmic reticulum is associated with an autophagic response. Am J Physiol Gastrointest Liver Physiol. 2000;279(5):G961-G974.

20. Kamimoto $\mathrm{T}$, et al. Intracellular inclusions containing mutant alpha1-antitrypsin $\mathrm{Z}$ are propagated in the absence of autophagic activity. J Biol Chem. 2006;281(7):4467-4476.

21. Teckman JH, An JK, Blomenkamp K, Schmidt B, Perlmutter D. Mitochondrial autophagy and injury in the liver in alpha 1-antitrypsin deficiency. Am J Physiol Gastrointest Liver Physiol. 2004;286(5):G851-G862.

22. Feldmann G, et al. The ultrastructure of hepatocytes in alpha-1-antitrypsin deficiency with the genotype Pi--. Gut. 1975;16(10):796-799.

23. Setten RL, Rossi JJ, Han SP. The current state and future directions of RNAi-based therapeutics. Nat Rev Drug Discov. 2019;18(6):421-446.

24. Wooddell CI, et al. Hepatocyte-targeted RNAi therapeutics for the treatment of chronic hepatitis B virus infection. Mol Ther. 2013;21(5):973-985.

25. Turner AM, et al. Hepatic-targeted RNA interference provides robust and persistent knockdown of alpha-1 antitrypsin levels in ZZ patients. J Hepatol. 2018;69(2):378-384

26. An JK, Blomenkamp K, Lindblad D, Teckman JH. Quantitative isolation of alphalAT mutant Z protein polymers from human and mouse livers and the effect of heat. Hepatology. 2005;41(1):160-167.

27. Blomenkamp KS, Teckman JH. Semiquantitation of monomer and polymer alpha-1 antitrypsin by centrifugal separation and assay by western blot of soluble and insoluble components. Methods Mol Biol. 2017;1639:227-234.

28. Geller SA, Nichols WS, Dycaico MJ, Felts KA, Sorge JA. Histopathology of alpha 1-antitrypsin liver disease in a transgenic mouse model. Hepatology. 1990;12(1):40-47.

29. Giovannoni I, Callea F, Stefanelli M, Mariani R, Santorelli FM, Francalanci P. Alpha-1-antitrypsin deficiency: from genoma to liver disease. PiZ mouse as model for the development of liver pathology in human. Liver Int. 2015;35(1):198-206.

30. Marcus NY, Blomenkamp K, Ahmad M, Teckman JH. Oxidative stress contributes to liver damage in a murine model of alpha-1-antitrypsin deficiency. Exp Biol Med (Maywood). 2012;237(10):1163-1172.

31. Li B, et al. The melanoma-associated transmembrane glycoprotein Gpnmb controls trafficking of cellular debris for degradation and is essential for tissue repair. FASEB J. 2010;24(12):4767-4781.

32. Snider NT, et al. Energy determinants GAPDH and NDPK act as genetic modifiers for hepatocyte inclusion formation. $J$ Cell Biol. 2011;195(2):217-229.

33. Fromenty B, Berson A, Pessayre D. Microvesicular steatosis and steatohepatitis: role of mitochondrial dysfunction and lipid peroxidation. J Hepatol. 1997;26(suppl 1):13-22.

34. Sakamoto M, Hirohashi S, Shimosato Y. Early stages of multistep hepatocarcinogenesis: adenomatous hyperplasia and early hepatocellular carcinoma. Hum Pathol. 1991;22(2):172-178.

35. Ott M, Gogvadze V, Orrenius S, Zhivotovsky B. Mitochondria, oxidative stress and cell death. Apoptosis. 2007;12(5):913-922.

36. Larsson C. Natural history and life expectancy in severe alpha1-antitrypsin deficiency, Pi Z. Acta Med Scand. 1978;204(5):345-351.

37. Dawwas MF, Davies SE, Griffiths WJ, Lomas DA, Alexander GJ. Prevalence and risk factors for liver involvement in individuals with PiZZ-related lung disease. Am J Respir Crit Care Med. 2013;187(5):502-508

38. Wang L, et al. Alpha-1 antitrypsin deficiency liver disease, mutational homogeneity modulated by epigenetic heterogeneity with links to obesity. Hepatology. 2019;70(1):51-66.

39. Greten FR, Grivennikov SI. Inflammation and cancer: triggers, mechanisms, and consequences. Immunity. 2019;51(1):27-41.

40. Benedetti A, Jézéquel AM, Orlandi F. Preferential distribution of apoptotic bodies in acinar zone 3 of normal human and rat liver. J Hepatol. 1988;7(3):319-324.

41. Gooptu B, Ekeowa UI, Lomas DA. Mechanisms of emphysema in alpha1-antitrypsin deficiency: molecular and cellular insights. Eur Respir J. 2009;34(2):475-488.

42. Trubetskoy VS, et al. Phosphorylation-specific status of RNAi triggers in pharmacokinetic and biodistribution analyses. Nucleic Acids Res. 2017;45(3):1469-1478.

43. Livak KJ, Schmittgen TD. Analysis of relative gene expression data using real-time quantitative PCR and the 2(-Delta Delta C(T)) method. Methods. 2001;25(4):402-408

44. Bozzola JJ, Russell, LD. Electron Microscopy: Principles and Techniques for Biologists. Sudbury, Massachusetts, USA: Jones and Bartlett Publishers; 1999. 